\title{
Stability of planar nonautonomous dynamic systems
}

\section{Gro Hovhannisyan*}

\section{"Correspondence:} ghovhann@kent.edu Kent State University at Stark, 6000 Frank Ave. NW, Canton, $\mathrm{OH}$ 44720-7599, USA

\begin{abstract}
We are describing the stable nonautonomous planar dynamic systems with complex coefficients by using the asymptotic solutions (phase functions) of the characteristic (Riccati) equation. In the case of nonautonomous dynamic systems, this approach is more accurate than the eigenvalue method. We are giving a new construction of the energy (Lyapunov) function via phase functions. Using this energy, we are proving new stability and instability theorems in terms of the characteristic function that depends on unknown phase functions. By different choices of the phase functions, we deduce stability theorems in terms of the auxiliary function of coefficients $R A(t)$, which is invariant with respect to the lower triangular transformations. We discuss some examples and compare our theorems with the previous results.
\end{abstract}

MSC: $34 \mathrm{D} 20$

Keywords: nonautonomous dynamic system; stability; attractivity to the origin; asymptotic stability; asymptotic solutions; characteristic function; Lyapunov function; energy function

\section{Introduction}

We are interested in the behavior of a given solution $u(t)$ of the nonlinear planar dynamic system

$$
u^{\prime}(t)=A(t, u) u(t), \quad A(t, u)=\left(\begin{array}{ll}
a_{11}(t, u(t)) & a_{12}(t, u(t)) \\
a_{21}(t, u(t)) & a_{22}(t, u(t))
\end{array}\right), \quad t \geq T
$$

where $a_{k j}(t, u(t))$ are complex-valued functions from $C^{2}(T, \infty)$, and $u(t)=\left(\begin{array}{l}u_{1}(t) \\ u_{2}(t)\end{array}\right)$. Since we are assuming that the solution $u(t)$ of (1.1) is given (fixed), system (1.1) may be considered as a linear nonautonomous system with coefficients $A(t)=A(t, u)$ depending only on a time variable.

Here and further, $C^{k}(T, \infty)$ is the set of $k$ times differentiable functions on $(T, \infty)$, $L_{1}(T, \infty)$ is the set of Lebesgue absolutely integrable functions on $(T, \infty)$, and $\mathrm{BV}(T, \infty)$ is the set of functions of bounded variation on $(T, \infty)$.

Dynamic system (1.1) is said to be stable if for any $\varepsilon>0$ and for any solution $u(t)$ of (1.1) there exists $\delta(T, \varepsilon)>0$ such that $\|u(t)\|<\varepsilon$ for all $t \geq T$, whenever $\|u(T)\|=$ $\sqrt{\left|u_{1}(T)\right|^{2}+\left|u_{2}(T)\right|^{2}}<\delta(T, \varepsilon)$. Dynamic system (1.1) is said to be attractive (to the origin) if for every solution $u(t)$ of $(1.1)$

$$
\lim _{t \rightarrow \infty} u(t)=0 .
$$


Dynamic system (1.1) is asymptotically stable if it is stable and attractive.

A solution $u(t)$ of $(1.1)$ is stable if for any $\varepsilon>0$ there exists $\delta(T, \varepsilon)>0$ such that $\|u(t)\|<\varepsilon$ for all $t \geq T$, whenever $\|u(T)\|=\sqrt{\left|u_{1}(T)\right|^{2}+\left|u_{2}(T)\right|^{2}}<\delta(T, \varepsilon)$.

A solution of (1.1) $u(t)$ is asymptotically stable (attractive to the origin) if (1.2) is true.

It is well-known that for a nonautonomous system with the complex eigenvalues $\lambda_{j}(t)$, $j=1, \ldots, n$, the classical Routh-Hurvitz condition of stability $\operatorname{Re}\left[\lambda_{j}\right] \leq 0, j=1, \ldots, n$, fails. Indeed, nonautonomous system (1.1) with

$$
A(t)=\left(\begin{array}{cc}
\lambda_{1} & e^{t \mu} \\
0 & \lambda_{2}
\end{array}\right), \quad \operatorname{Re}\left[\lambda_{1}\right] \leq 0, \operatorname{Re}\left[\lambda_{2}\right] \leq 0
$$

is unstable if $\operatorname{Re}[\mu]>-\operatorname{Re}\left[\lambda_{2}\right]$, although the Routh-Hurvitz condition is satisfied. Necessary and sufficient conditions of asymptotic stability of this system,

$$
\operatorname{Re}\left[\lambda_{1}\right]<0, \quad \operatorname{Re}\left[\lambda_{2}\right]<0, \quad \operatorname{Re}\left[\lambda_{2}\right]<-\operatorname{Re}[\mu],
$$

could be found from the explicit solutions

$$
u_{1}(t)=\frac{C_{2} e^{t\left(\mu+\lambda_{2}\right)}}{\mu+\lambda_{2}-\lambda_{1}}+C_{1} e^{t \lambda_{1}}, \quad u_{2}(t)=C_{2} e^{t \lambda_{2}}
$$

This example shows that the description of stability of nonautonomous dynamic systems in terms of the eigenvalues is not accurate.

The usual method of investigation of asymptotic stability of differential equations is the Lyapunov direct method that uses energy functions and Lyapunov stability theorems [13].

The asymptotic representation of solutions and error estimates in terms of the characteristic function was used in [4-6] to prove asymptotic stability. In this paper we describe the stable dynamic systems by using energy approach with the use of the characteristic function (see (1.7) below), which is a more accurate tool than the eigenvalues.

The main idea of this paper is to construct the energy function in such a way that the time derivative of this energy is the linear combination of the characteristic functions (see (2.15) below). Using this energy, we prove main stability theorems for two-dimensional systems in terms of unknown phase functions (see Theorems 3.1-3.3).

Theorems 3.1-3.3 are similar to Lyapunov stability theorems with additional construction of an energy function in terms of the phase functions. Theorems 3.1-3.3 are applicable to a wide range of nonlinear systems with complex-valued coefficients (see Example 5.2 below or the linear Dirac equation with complex coefficients in [7]) since they have the flexibility in the choice of an energy function.

To show that our theorems are useful, we deduce different versions of stability theorems (old well-known and some new ones) by using different phase functions as asymptotic solutions of the characteristic equation (see (2.8) below). Moreover, we formulate some of the conditions of stability in terms of the auxiliary function $R A(t)$ (see (2.10) below), which is invariant with respect to the lower triangular transformations (see Theorem A.1). Note that there is no universal stability theorem in terms of coefficients for nonautonomous system (1.1) since there is no universal formula for an asymptotic solution of the characteristic equation. 
As an application (see Example 5.5), we prove the asymptotic stability of the nonlinear Matukuma equation from astrophysics $[8,9]$.

Consider the second-order linear equation

$$
L[v]=v^{\prime \prime}(t)+2 P(t) v^{\prime}(t)+Q(t) v(t)=0 .
$$

Define the characteristic (Riccati) equation of (1.6)

$$
C L_{j}(t)=e^{-\int_{T}^{t} \chi_{j}(s) d s} L\left(e^{\int_{T}^{t} \chi_{j}(s) d s}\right)=\chi_{j}^{\prime}(t)+\chi_{j}^{2}(t)+2 P(t) \chi_{j}(t)+Q(t)=0,
$$

where $C L_{j}(t)$ is said to be the characteristic function, and $\chi_{1,2}(t)$ are the phase functions. In Section 6 (see Lemma 6.1) the following lemma is proved.

Lemma 1.1 Assume that every solution $v(t) \in C^{2}(T, \infty)$ of $(1.6)$ approaches zero as $t \rightarrow \infty$, then

$$
\lim _{t \rightarrow \infty} \int_{T}^{t} \Re\left[2 P(s)+\frac{\chi_{1}^{\prime}(s)-\chi_{2}^{\prime}(s)}{\chi_{1}(s)-\chi_{2}(s)}\right] d s=\infty
$$

where $\chi_{1,2}(t) \in C^{1}(T, \infty)$ are solutions of characteristic equation (1.7).

In the proof of Lemma 1.1, it is shown that (1.8) is also a sufficient condition of attractivity of solutions of (1.6) to the origin under additional condition

$$
-\ln C \leq \int_{T}^{\infty}\left(\Re\left[\chi_{1}(s)-\chi_{2}(s)\right]\right) d s \leq \ln C, \quad C=\text { const }>0 .
$$

If the asymptotic behavior of $\chi_{1}(t)-\chi_{2}(t)$ as $t \rightarrow \infty$ is known, then the condition of attractivity (1.8) could be clarified. Unfortunately, there is no a simple formula for asymptotic behavior of $\chi_{1}(t)-\chi_{2}(t)$ depending on the behavior of $P(t), Q(t)$ as $t \rightarrow \infty$. Anyway, under some restrictions, one can obtain stability theorems for (1.6) by considering different asymptotic expansions of $\chi_{1}(t)-\chi_{2}(t)$.

Assume that for some positive constants $P_{1}, Q_{0}, Q_{m}$,

$$
\left|Q^{\prime}(t)\right|+|P(t)| \leq P_{1}, \quad Q_{0} \leq Q(t) \leq Q_{m}
$$

Theorem 1.2 (Ignatyev [10]) Suppose that the functions $P(t) \in C(T, \infty), Q(t) \in C^{1}(T, \infty)$ are real, and they satisfy conditions (1.10) and

$$
2 P(t)+\frac{Q^{\prime}(t)}{2 Q(t)} \geq m>0 \quad \text { for some } m=\text { const }
$$

Then linear equation (1.6) is asymptotically stable.

Condition that $\left|Q^{\prime}(t)\right|$ is bounded above in (1.10) was removed in [11].

Note that if

$$
\frac{\chi_{1}^{\prime}(t)-\chi_{2}^{\prime}(t)}{\chi_{1}(t)-\chi_{2}(t)}-\frac{Q^{\prime}(t)}{2 Q(t)} \in L_{1}(T, \infty)
$$


then condition (1.8) turns to

$$
\int_{T}^{\infty} \Re\left[2 P(s)+\frac{Q^{\prime}(t)}{2 Q(t)}\right] d s=\infty,
$$

and is an integral version of (1.11).

In [12] Ballieu and Peiffer introduced a more general condition than Ignatyev's one (1.11) for the attractivity (see (1.15), (1.16) below) of a nonlinear second-order equation.

Theorem 1.3 (Pucci-Serrin [9], Theorem B) Suppose that functions $P(t) \in C(T, \infty)$, $Q(t) \in C^{1}(T, \infty)$ are real, and there exists a non-negative continuous function $k(t)$ of bounded variation on $(T, \infty)$ such that

$$
\begin{aligned}
& v f(v)>0 \quad \text { for } v \neq 0, \\
& 2 P(t)+\frac{Q^{\prime}(t)}{2 Q(t)} \geq k(t) \sqrt{Q(t)}, \quad t \geq T, \\
& \int_{T}^{\infty} k(t) \sqrt{Q(t)} d t=\infty, \\
& \lim _{t \rightarrow \infty} \inf \left(\frac{\int_{T}^{t} k^{2}(s)\left[2 P(s)+\frac{Q^{\prime}(s)}{2 Q(s)}\right] d s}{\int_{T}^{t} k(s) \sqrt{Q(s)} d s}\right)<\infty,
\end{aligned}
$$

then every bounded solution of the nonlinear equation

$$
v^{\prime \prime}(t)+2 P(t) v^{\prime}(t)+Q(t) f(v)=0, \quad t \geq T,
$$

tends to zero as $t \rightarrow \infty$.

In this paper we prove general stability Theorems 3.1-3.3 in terms of unknown phase functions. Using these theorems we derive the versions of stability theorem of Pucci-Serrin [9], Smith [13], and some new ones.

\section{Energy and some other auxiliary functions}

Assuming $a_{12}(t) \neq 0$, consider the following second-order nonlinear equation associated with system (1.1):

$$
L\left[u_{1}\right]=u_{1}^{\prime \prime}(t)+2 P(t, u) u_{1}^{\prime}(t)+Q(t, u) u_{1}(t)=0,
$$

where

$$
\begin{aligned}
& 2 P(t, u)=-\operatorname{Tr}(A(t))-\frac{a_{12}^{\prime}(t, u(t))}{a_{12}(t, u(t))}, \quad Q(t, u)=\operatorname{det}(A(t))+\frac{W\left[a_{11}, a_{12}\right]}{a_{12}(t, u(t))}, \\
& \operatorname{Tr}(A(t)) \equiv a_{11}(t, u(t))+a_{22}(t, u(t)), \\
& \operatorname{det}(A(t)) \equiv a_{11}(t, u(t)) a_{22}(t, u(t))-a_{12}(t, u(t)) a_{21}(t, u(t)), \\
& W\left[a_{11}, a_{12}\right] \equiv a_{11}(t, u(t)) a_{12}^{\prime}(t, u(t))-a_{11}^{\prime}(t, u(t)) a_{12}(t, u(t)) .
\end{aligned}
$$


Remark 2.1 Note that using equation (1.1), one can eliminate dependence $a_{12}^{\prime}(t, u(t))$ on $u^{\prime}(t)$. Indeed $a_{12}^{\prime}(t, u(t))=\frac{\partial a_{12}}{\partial t}+\sum_{j=1}^{2} \frac{\partial a_{12}}{\partial u_{j}} \frac{\partial u_{j}(t)}{\partial t}=\frac{\partial a_{12}}{\partial t}+\sum_{j=1}^{2} \frac{\partial a_{12}}{\partial u_{j}}\left(a_{j 1} u_{1}+a_{j 2} u_{2}\right)$. Similar calculations show that $a_{12}^{\prime \prime}(t, u(t))$ depends only on $t, u(t)$, coefficients $a_{k j}(t, u(t))$, and their derivatives.

Here and further, often we suppress the dependence on $t$ and $u(t)$ for simplicity.

Introduce the characteristic function of (2.1) that depends on an unknown phase function $\theta_{j}(t)$ :

$$
C L_{j}(t)=C L\left(\theta_{j}\right)=\frac{L\left[e_{\theta_{j}}(t)\right]}{e_{\theta_{j}}(t)}=\theta_{j}^{\prime}(t)+\theta_{j}^{2}(t)+2 P(t, u) \theta_{j}(t)+Q(t, u),
$$

and the auxiliary function:

$$
H L(t)=\frac{C L_{1}(t)-C L_{2}(t)}{\theta_{1}(t)-\theta_{2}(t)}=\theta_{1}(t)+\theta_{2}(t)+\frac{\theta^{\prime}(t)}{\theta(t)}+2 P(t, u),
$$

where

$$
e_{\theta_{j}}(t)=e^{\int_{T}^{t} \theta_{j}(s) d s}, \quad j=1,2, \quad \theta(t) \equiv \frac{\theta_{1}(t)-\theta_{2}(t)}{2}
$$

Define the characteristic (Riccati) function of system (1.1)

$$
\begin{aligned}
C A_{j}(t) & =C A\left(\theta_{j}\right) \\
& =\theta_{j}^{\prime}(t)+\theta_{j}^{2}(t)-\theta_{j}(t)\left[\operatorname{Tr}(A(t))+\frac{a_{12}^{\prime}(t)}{a_{12}(t)}\right]+\operatorname{det}(A(t))+\frac{W\left[a_{11}, a_{12}\right]}{a_{12}(t)} .
\end{aligned}
$$

Equation $C A_{j}(t)=0$ is the characteristic equation of system (1.1). For diagonal system (1.1), formulas (2.8) fail (for this case, see (A.23)).

Introduce the auxiliary functions

$$
\begin{aligned}
& H A(t)=\frac{C A_{1}(t)-C A_{2}(t)}{\left(\theta_{1}(t)-\theta_{2}(t)\right)}=\frac{\theta_{1}^{\prime}(t)-\theta_{2}^{\prime}(t)}{\theta_{1}(t)-\theta_{2}(t)}+\theta_{1}(t)+\theta_{2}(t)-\operatorname{Tr}(A(t))-\frac{a_{12}^{\prime}(t)}{a_{12}(t)}, \\
& R A(t)=\operatorname{det}(A(t))-\frac{(\operatorname{Tr} A(t))^{2}}{4}+\frac{W\left[t, a_{11}-a_{22}, a_{12}\right]}{2 a_{12}(t)}+\frac{a_{12}^{\prime \prime}(t)}{2 a_{12}(t)}-\frac{3 a_{12}^{\prime 2}(t)}{4 a_{12}^{2}(t)} .
\end{aligned}
$$

To explain the motivation for the choice of an energy function for system (1.1) (assuming $\left.a_{12}(t, u) \neq 0\right)$, consider a representation of solutions of (1.1) in Euler form (see [6]):

$$
u_{1}=C_{1} e_{\chi_{1}}(t)+C_{2} e_{\chi_{2}}(t), \quad u_{2}=C_{1} U_{1}(t) e_{\chi_{1}}(t)+C_{2} U_{2}(t) e_{\chi_{2}}(t)
$$

where $\chi_{j}(t), j=1,2$, are exact solutions of the characteristic equation $C A_{j}\left(\chi_{j}\right)=0, e_{\chi_{j}}(t)$ are defined as in (2.7), and

$$
U_{j}(t)=\frac{\chi_{j}(t)-a_{11}(t, u(t))}{a_{12}(t, u(t))}, \quad j=1,2 .
$$

For the case of linear system (1.1), representation (2.11) gives the general solution of (1.1), where $C_{1}, C_{2}$ are constants. For a nonlinear system, $C_{1}, C_{2}$ depend on a solution $u(t)$. 
Solving equations (2.11) for $C_{1,2}$, we get

$$
C_{1}=\frac{a_{12} u_{2}-\left(\chi_{2}-a_{11}\right) u_{1}}{\left(\chi_{1}-\chi_{2}\right) e_{\chi_{1}}(t)}, \quad C_{2}=-\frac{a_{12} u_{2}-\left(\chi_{1}-a_{11}\right) u_{1}}{\left(\chi_{1}-\chi_{2}\right) e_{\chi_{2}}(t)}
$$

Replacing $\chi_{j}(t)$ by arbitrary differentiable functions $\theta_{j}(t)$, we define auxiliary energy functions

$$
E_{j}(t)=E_{j}\left(\theta_{j}(t)\right)=\left|C_{j}\right|^{2}=\frac{\left|a_{12} u_{2}-\left(\theta_{j}-a_{11}\right) u_{1}\right|^{2}}{\left|\left(\theta_{1}-\theta_{2}\right) e_{\theta_{3-j}}\right|^{2}}, \quad j=1,2
$$

Remark 2.2 Although (2.14) are not constants for a nonlinear or nonautonomous system, they are useful for the study of stability. One can expect that for an appropriate choice of $\theta_{j}(t)$ these energy functions are approximately conservative expressions for some nonlinear systems that are close to linear.

The derivative of the energy functions (2.14) may be written (see (6.23) below) as a linear combination of the characteristic functions:

$$
E_{j}^{\prime}(t)=\frac{2 \Re\left[\overline{\left(\theta_{j}-a_{11}\right)}\left|u_{1}\right|^{2} C A_{j}-\overline{u_{2} a_{12}} u_{1} C A_{j}-H A\left|\left(\theta_{j}-a_{11}\right) u_{1}-a_{12} u_{2}\right|^{2}\right]}{\left|\theta e_{\theta_{3-j}}\right|^{2}} .
$$

From (2.15) it follows that if for any given solution $u(t)$ of $(1.1)$ the phase functions $\theta_{j}(t)$ satisfy characteristic equation, that is, $\theta_{j}(t)=\chi_{j}(t), j=1,2$, then energy conservation laws $E_{j}(t)=$ const, $j=1,2$ are satisfied.

Otherwise, (2.15) means that the error of asymptotic solutions is measured by the characteristic function.

Define (total) energy function as a non-negative quadratic form

$$
E(t)=E_{1}(t)+E_{2}(t)
$$

Remark 2.3 If the phase functions are chosen as

$$
\theta_{1,2}(t)= \pm \theta(t)-\frac{\theta^{\prime}(t)}{2 \theta(t)}+\frac{\operatorname{Tr}(A(t))}{2}+\frac{a_{12}^{\prime}(t)}{2 a_{12}(t)}
$$

where $\theta(t)$ is an arbitrary differentiable function, then

$$
H A(t) \equiv 0
$$

\section{Stability theorems in terms of unknown phase functions}

In this section we formulate the main Theorems 3.1-3.3 of the paper.

Theorem 3.1 Suppose that for a solution $u(t)$ of $(1.1)$, we have $A(t, u) \in C^{1}(T, \infty)$, and there exist the complex-valued functions $p_{1}(t), p_{2}(t) ; \theta_{1}(t), \theta_{2}(t) \in C^{1}(T, \infty)$ and the real numbers $c>0, \alpha$ such that for all $t \geq T$ we have $a_{12}(t, u) \neq 0$ and

$$
\left|\frac{\theta_{1}(t)-a_{11}(t, u)}{a_{12}(t, u)}\right|^{2}+\left|\frac{\theta_{2}(t)-a_{11}(t, u)}{a_{12}(t, u)} e_{-s}(t)\right|^{2}+1+\left|e_{-s}(t)\right|^{2} \leq c|\theta(t)|^{2 \alpha},
$$




$$
\begin{aligned}
& \left(1+\left|e_{s}(t)\right|^{2}\right) \Re\left[J_{0}(t)+H A(t)\right]>\left(1-\left|e_{s}(t)\right|^{2}\right) \Re\left[p_{1}(t)-p_{2}(t)\right] \\
& \int_{T}^{\infty} J(t, u(t)) d t \leq c<\infty
\end{aligned}
$$

where $s(t)=\theta_{1}(t)-\theta_{2}(t)-p_{1}(t)+p_{2}(t), J(t, u(t))=J_{1}(t), 2 \theta(t) \equiv \theta_{1}(t)-\theta_{2}(t)$

$$
\begin{aligned}
& J_{1}(t)=\Re\left[2 \theta(t)+\frac{(2 \alpha-1) \theta^{\prime}(t)}{\theta(t)}+\operatorname{Tr}(A(t))+\frac{a_{12}^{\prime}(t, u)}{a_{12}(t, u)}+p_{2}(t)-p_{1}(t)\right]+J_{0}(t), \\
& J_{0}(t)=\sqrt{\mid \frac{C A_{1}(t)\left|e_{s}(t)\right|}{\theta_{1}(t)-\theta_{2}(t)}-\frac{\overline{C A_{2}(t)}\left|e_{-s}(t)\right|}{\left.\overline{\theta_{1}(t)-\theta_{2}(t)}\right|^{2}+\left[\Re\left(p_{2}(t)-p_{1}(t)+\frac{C A_{1}(t)+C A_{2}(t)}{\theta_{1}(t)-\theta_{2}(t)}\right)\right]^{2}} .}
\end{aligned}
$$

Then the solution $u(t)$ of system (1.1) is stable.

Remark 3.1 Since stability conditions (3.1)-(3.3) of Theorem 3.1 are given in terms of estimates with constants that depend on solutions of (1.1), system (1.1) is stable if these estimates are satisfied uniformly for all solutions (with constants that do not depend on solutions).

Remark 3.2 Note that for a linear nonautonomus system (1.1) with the choice $\theta_{j}(t)=\chi_{j}(t)$, $j=1,2, \mathfrak{R}\left[p_{2}(t)-p_{1}(t)\right] \equiv 0$, the error function $J_{0}(t) \equiv 0$ and conditions (3.1), (3.3) are close to the necessary and sufficient condition of the stability.

Theorem 3.2 Suppose that for a solution $u(t)$ of $(1.1) A(t, u) \in C^{1}(T, \infty)$, there exist the complex-valued functions $p_{1}(t), p_{2}(t) ; \theta_{1}(t), \theta_{2}(t) \in C^{1}(T, \infty)$, and the real numbers $c>0, \alpha$ such that for all $t \geq T, a_{12}(t, u) \neq 0$ and conditions (3.1), (3.2),

$$
\int_{T}^{\infty} J(t, u(t)) d t=-\infty
$$

are satisfied with $J(t, u(t))=J_{1}(t)$ as in (3.4), (3.5).

Then the solution $u(t)$ of system (1.1) is asymptotically stable.

Theorem 3.3 Suppose that for a solution $u(t)$ of $(1.1)$, we have $A(t, u) \in C^{1}(T, \infty)$, and there exist the complex-valued functions $p_{1}(t), p_{2}(t) ; \theta_{1}(t), \theta_{2}(t) \in C^{1}(T, \infty)$ such that for all $t \geq T$ we have $a_{12}(t, u) \neq 0$,

$$
\begin{aligned}
& \Re[H A(t)]<J_{0}(t)-\left|\Re\left[p_{1}(t)-p_{2}(t)\right]\right|, \\
& \lim _{t \rightarrow \infty} \frac{\exp \int_{T}^{t} J_{2}(s, u) d s}{\left|\left(\theta_{1}-a_{11}\right) e_{s}\right|^{2}+\left|\theta_{2}-a_{11}\right|^{2}+\left|a_{12}\right|^{2}\left(1+\left|e_{s}\right|^{2}\right)(t)}=\infty,
\end{aligned}
$$

where $J_{0}(t)$ is defined in (3.5), and

$$
J_{2}(t, u)=\Re\left[\theta_{1}(t)-\theta_{2}(t)+p_{2}(t)-p_{1}(t)+\frac{\theta^{\prime}(t)}{\theta(t)}+\operatorname{Tr}(A(t))+\frac{a_{12}^{\prime}(t)}{a_{12}(t)}\right]-J_{0}(t) .
$$

Then the solution $u(t)$ of system (1.1) is unstable. 
Example 3.1 From Theorem 3.3 it follows that the linear canonical equation

$$
v^{\prime \prime}(t)+2 b t^{\gamma-1} v^{\prime}(t)+c t^{2 \beta-2} v(t)=0, \quad c>0, b<0, \beta>\gamma>0
$$

is unstable.

Remark 3.3 If

$$
\operatorname{Re}\left[\theta_{1}(t)-\theta_{2}(t)\right] \geq \operatorname{Re}\left[p_{1}(t)-p_{2}(t)\right] \geq 0,
$$

then $\operatorname{Re}[s] \geq 0,\left|e_{s}(t)\right| \geq 1$ and condition (3.2) is satisfied if $\operatorname{Re}\left[H A(t)+J_{0}(t)\right]>0$.

Otherwise (3.2) is satisfied if $J_{0}(t)>0, \operatorname{Re}[H A] \geq\left|\operatorname{Re}\left[p_{1}(t)-p_{2}(t)\right]\right|$.

Under condition (3.11), condition (3.1) turns to

$$
\left|\theta_{1}-a_{11}\right|^{2}+\left|\left(\theta_{2}-a_{11}\right) e_{-s}\right|^{2}+\left|a_{12} e_{-s}\right|^{2} \leq\left|a_{12}\right|^{2}\left(c\left|\theta_{1}-\theta_{2}\right|^{2 \alpha}-1\right),
$$

which is satisfied if

$$
\left|\theta_{1}-a_{11}\right|^{2}+\left|\left(\theta_{2}-a_{11}\right)\right|^{2} \leq\left|a_{12}\right|^{2}\left(c\left|\theta_{1}-\theta_{2}\right|^{2 \alpha}-2\right)
$$

or

$$
3\left|\theta_{1}(t)-a_{11}(t)\right|^{2}+2\left|\theta_{1}(t)-\theta_{2}(t)\right|^{2} \leq\left|a_{12}\right|^{2}\left(c\left|\theta_{1}(t)-\theta_{2}(t)\right|^{2 \alpha}-2\right) .
$$

Sometimes it is convenient to use other than (3.4) formula for $J_{1}(t)$ :

$$
J_{1}(t)=\Re\left[2 \theta_{1}(t)+\frac{2 \alpha \theta^{\prime}(t)}{\theta(t)}-H A(t)+p_{2}(t)-p_{1}(t)\right]+J_{0}(t) .
$$

Remark 3.4 If $p_{1}(t) \equiv p_{2}(t) \equiv 0$, and there exists a function $\theta(t) \in C^{1}(T, \infty)$ such that

$$
\int_{T}^{\infty}\left|\frac{e_{ \pm 2 \theta}(t) C A_{j}(t)}{\theta(t)}\right| d t<\infty, \quad j=1,2
$$

then $H A(t) \in L_{1}(T, \infty), J_{0}(t) \in L_{1}(T, \infty)$. In this case formula (3.5) is simplified

$$
J_{0}(t)=\sqrt{(\Re[H A(t)])^{2}+\left|\frac{C A_{1}(t)\left|e_{s}(t)\right|+C A_{2}(t)\left|e_{-s}(t)\right|}{\theta_{1}(t, u)-\theta_{2}(t, u)}\right|^{2}},
$$

and we get $\operatorname{Re}\left[J_{0}+H A\right] \geq 0$. From Theorem 3.1 it follows that in this case the solution $u(t)$ of system (1.1) is asymptotically stable if for some real numbers $\alpha, l$

$$
\Re\left[\theta_{1}(t)+\frac{\alpha \theta^{\prime}(t)}{\theta(t)}\right] \leq l<0, \quad t \geq T
$$

are satisfied (see (3.13), (3.6)). 
Note that (3.16) is a nonautonomous analogue of the classical asymptotic stability criterion of Routh-Hurvitz.

If the phase functions $\theta_{1,2}$ are chosen by formula (2.17), then $H A(t) \equiv 0$, and

$$
J_{1}(t)=\Re\left[2 \theta_{1}(t)+\frac{2 \alpha \theta^{\prime}(t)}{\theta(t)}+p_{2}-p_{1}\right]+\left|\frac{C A_{1}(t)\left|e_{2 \theta}(t)\right|+C A_{1}(t)\left|e_{-2 \theta}(t)\right|}{2 \theta(t)}\right| .
$$

From Theorems 3.1-3.3 one can deduce stability theorems for second-order equation (2.1). The attractivity to the origin for the solution of equation (2.1) is valid even by removing condition (3.1) (compare Theorem 3.2 with the following theorem).

Theorem 3.4 Suppose that for a given solution $u_{1}(t)$ of $(2.1)$, there exist the complex-valued functions $p_{1}, p_{2} ; \theta_{1,2} \in C^{1}(T, \infty)$ such that conditions (3.2), (3.6) are satisfied with $J(t, u)=$ $J_{3}(t)$ defined as

$$
\begin{aligned}
& J_{3}(t)=\Re\left[\theta_{1}(t)-\theta_{2}(t)-\frac{\theta^{\prime}(t)}{\theta(t)}-2 P(t, u)+p_{2}(t)-p_{1}(t)\right]+J_{0}(t), \\
& J_{0}(t)=\sqrt{\left|\frac{C L_{1}\left|e_{s}\right|}{\theta_{1}-\theta_{2}}-\frac{\overline{C L_{2}}\left|e_{-s}\right|}{\overline{\theta_{1}-\theta_{2}}}\right|^{2}+\left[\Re\left(p_{2}-p_{1}+\frac{C L_{1}+C L_{2}}{\theta_{1}-\theta_{2}}\right)\right]^{2}} .
\end{aligned}
$$

Then the solution $u_{1}(t)$ of $(2.1)$ approaches zero as $t \rightarrow \infty$.

\section{Choosing}

$$
p_{1}(t) \equiv 2 \theta(t), \quad p_{2}(t) \equiv 0, \quad \alpha=1
$$

from Theorem 3.1 (in view of $s=0$ ), we obtain the following theorem.

Theorem 3.5 Suppose that for a given solution $u(t)$ of $(1.1), A(t, u) \in C^{1}(T, \infty)$, and there exist complex-valued functions $\theta_{1}(t), \theta_{2}(t) \in C^{1}(T, \infty)$ such that for all $t \geq T$ we have $a_{12}(t, u) \neq 0$,

$$
\begin{aligned}
& \left|\theta_{1}(t)-a_{11}(t, u)\right|^{2}+\left|\theta_{2}(t)-a_{11}(t, u)\right|^{2}+2\left|a_{12}(t, u)\right|^{2} \leq c\left|a_{12}(t, u)\right|^{2}|\theta(t)|^{2}, \\
& J_{0}(t)+\Re[H A(t)]=J_{0}(t)+\Re\left[\theta_{1}(t)+\theta_{2}(t)+\frac{\theta^{\prime}(t)}{\theta(t)}-\operatorname{Tr}(A)-\frac{a_{12}^{\prime}(t, u)}{a_{12}(t, u)}\right] \geq 0,
\end{aligned}
$$

and (3.6) are satisfied, where $J(t, u)=J_{4}(t)$,

$$
\begin{aligned}
& J_{4}(t)=\Re\left(\frac{\theta^{\prime}(t)}{\theta(t)}+\operatorname{Tr}(A(t))+\frac{a_{12}^{\prime}(t, u)}{a_{12}(t, u)}+J_{0}(t)\right), \\
& J_{0}(t)=\sqrt{\left|\frac{C A_{1}(t)}{2 \theta(t)}-\frac{\overline{C A_{2}(t)}}{2 \overline{\theta(t)}}\right|^{2}+\left[\Re\left(\frac{C A_{1}(t)+C A_{2}(t)}{2 \theta(t)}-2 \theta(t)\right)\right]^{2}} .
\end{aligned}
$$

Then the solution $u(t)$ of system (1.1) is asymptotically stable.

By choosing

$$
\alpha=0, \quad p_{1}(t)=p_{2}(t)=0,
$$


we have $s(t)=2 \theta(t)$, and assuming (3.11) we get $\left|e_{s}(t)\right| \geq 1$. From Theorem 3.2 we deduce the following theorem.

Theorem 3.6 Suppose that for a given solution $u(t)$ of $(1.1), A(t, u) \in C^{1}(T, \infty)$, and there exist complex-valued functions $\theta_{1}(t), \theta_{2}(t) \in C^{1}(T, \infty)$ such that for all $t \geq T$ we have $a_{12}(t, u) \neq 0$,

$$
\begin{aligned}
& \Re\left[\theta_{1}(t)-\theta_{2}(t)\right] \geq 0, \quad\left|\theta_{1}(t)-a_{11}(t, u)\right|^{2}+\left|\theta_{2}(t)-a_{11}(t, u)\right|^{2} \leq C\left|a_{12}(t, u)\right|^{2}, \\
& J_{0}(t)+\Re[H A(t)]=J_{0}(t)+\Re\left[\frac{\theta^{\prime}(t)}{\theta(t)}+\theta_{1}(t)+\theta_{2}(t)-\operatorname{Tr}(A(t))-\frac{a_{12}^{\prime}(t, u)}{a_{12}(t, u)}\right] \geq 0,
\end{aligned}
$$

and (3.6) are satisfied with $J_{0}(t)$ is as in (3.5), and $J(t, u)=J_{5}(t)$ :

$$
J_{5}(t)=J_{0}(t)+\Re\left[2 \theta_{1}(t)-H A(t)\right]
$$

Then the solution $u(t)$ of system (1.1) is asymptotically stable.

Theorem 3.7 Suppose that for a given solution $u(t)$ of $(1.1), A(t, u) \in C^{1}(T, \infty)$, there exist complex-valued function $\theta_{2}(t) \in L_{1}(T, t)$ and the real numbers $c>0, \alpha$ such that for all $t \geq T$ we have $a_{12}(t, u) \neq 0$ and the conditions

$$
\begin{aligned}
& \Re[\theta(t)] \geq 0, \quad 2 \theta(t) \equiv \frac{d}{d t} \ln \left(1+2 \theta(T) \int_{T}^{t} e^{\int_{T}^{s}\left(\operatorname{Tr} A(y)+\frac{a_{12}^{\prime}(y)}{a_{12}}-2 \theta_{2}(y)\right) d y} d s\right), \\
& |2 \theta(t)|^{2}+\left|2 \theta_{2}(t)-2 a_{11}(t, u)\right|^{2} \leq\left|a_{12}(t, u)\right|^{2}\left(c|\theta|^{2 \alpha}-1\right),
\end{aligned}
$$

equation (3.3) (or (3.6)) are satisfied, where $J(t, u(t))=J_{6}(t)$,

$$
J_{6}(t)=J_{0}(t)+\Re\left[2 \operatorname{Tr}(A(t))+\frac{2 a_{12}^{\prime}(t, u)}{a_{12}(t, u)}-2 \theta_{2}(t)+\frac{2(\alpha-1) \theta^{\prime}(t)}{\theta}\right]
$$

or

$$
\begin{aligned}
& J_{6}(t)=J_{0}(t)+\Re\left[2 \alpha\left(\operatorname{Tr}(A(t))+\frac{a_{12}^{\prime}(t, u)}{a_{12}(t, u)}-2 \theta_{2}(t)-2 \theta(t)\right)+4 \theta(t)+2 \theta_{2}(t)\right], \\
& J_{0}(t)=\left|C A_{2}(t)\right|\left[\frac{1+\left|1+2 \theta(T) \int_{T}^{t} e^{\int_{T}^{s}\left(\operatorname{Tr} A(y)+\frac{a_{12}^{\prime}(y)}{a_{12}}-2 \theta_{2}(y)\right) d y} d s\right|^{2}}{\left.2|\theta(T)| e^{\int_{T}^{t} \Re\left[\operatorname{Tr} A(y)+\frac{a_{12}^{\prime}(y)}{a_{12}}-2 \theta_{2}(y)\right] d y}\right] .}\right.
\end{aligned}
$$

Then the solution $u(t)$ of system (1.1) is stable (or asymptotically stable).

Theorem 3.8 Suppose that for a solution $u_{1}(t)$ of (2.1), $P(t, u) \in C^{1}(T, \infty), Q(t, u) \in$ $C(T, \infty)$, there exist the real numbers $c>0, \alpha$ and the complex-valued function $\theta_{2}(t) \in$ $L_{1}(T, t)$ such that for all $t \geq T$, conditions (3.29) and

$$
\int_{T}^{\infty} \Re\left[2 P(t, u)+\frac{\theta^{\prime}(t)}{\theta(t)}-2 \theta(t)-J_{0}(t)\right] d t=\int_{T}^{\infty} \Re\left[-2 \theta_{2}(t)-4 \theta(t)-J_{0}(t)\right] d t=\infty
$$

are satisfied, where $\theta(t), J_{0}(t)$ are given by (3.29), (3.32).

Then the solution $u_{1}(t)$ of equation (2.1) approaches zero as $t \rightarrow \infty$. 


\section{Stability of the planar dynamic systems}

From Theorems 3.1-3.3 one can deduce more useful asymptotic stability theorems in terms of coefficients of (1.1) by choosing the phase functions as asymptotic solutions of the characteristic equation.

Theorem 4.1 Suppose that for a solution $u(t)$ of $(1.1)$, we have $A(t, u) \in C^{3}(T, \infty)$, and for all $t \geq T$ the conditions

$$
\begin{aligned}
& \Re[s(t)] \geq 0, \quad \Re\left[p_{1}(t)-p_{2}(t)\right] \geq 0, \quad s(t)=2 \sqrt{-R A(t)}-p_{1}(t)+p_{2}(t), \\
& |R A(t)|+\left|\operatorname{Tr}(A(t))+\frac{a_{12}^{\prime}(t)}{a_{12}(t)}-2 a_{11}(t)\right|^{2} \leq\left|a_{12}(t)\right|^{2}\left(c|R A(t)|^{\alpha}-1\right),
\end{aligned}
$$

and (3.3) $\left(\right.$ or (3.6)) are satisfied, where $J(t, u(t))=J_{7}(t)$,

$$
\begin{aligned}
J_{7}(t)= & J_{0}(t)+\Re\left[2 \sqrt{-R A(t)}+\frac{(2 \alpha-1) R A^{\prime}(t, u)}{2 R A(t)}\right. \\
& \left.+\operatorname{Tr}(A(t))+\frac{a_{12}^{\prime}(t, u)}{a_{12}(t, u)}+p_{2}(t)-p_{1}(t)\right], \\
J_{0}(t)= & \sqrt{\left[\Re\left(\frac{R A^{\prime}(t, u)}{2 R A(t)}\right)\right]^{2}+\left(\operatorname{Re}\left[p_{2}-p_{1}\right]\right)^{2}(t)+\frac{\left|R A^{\prime}(t)\right|^{2}\left(\left|e_{s}(t)\right|-\left|e_{-s}(t)\right|\right)^{2}}{16|R A(t)|^{2}}} .
\end{aligned}
$$

Then the solution $u(t)$ of system (1.1) is stable (or asymptotically stable).

Theorem 4.2 Suppose that for a solution $u(t)$ of $(1.1)$, we have $A(t, u) \in C^{2}(T, \infty)$, and for all $t \geq T$ we have $a_{12}(t, u) \neq 0$ and

$$
\begin{aligned}
& \frac{1}{t^{2}}+\left|\operatorname{Tr}(A(t))+\frac{a_{12}^{\prime}(t, u)}{a_{12}(t, u)}-2 a_{11}(t, u)\right|^{2} \leq c\left|a_{12}(t, u)\right|^{2}, \\
& \int_{T}^{\infty} \Re\left[\frac{2}{t}+\operatorname{Tr}(A(t))+\frac{a_{12}^{\prime}(t, u)}{a_{12}(t, u)}+\frac{2 t^{2}}{T}|R A(t)|\right] d t=-\infty
\end{aligned}
$$

Then the solution $u(t)$ of system (1.1) is asymptotically stable.

Theorem 4.3 Suppose that for a solution $u(t)$ of $(1.1), A(t, u) \in C^{2}(T, \infty)$, for some numbers $c>0, \alpha$, and for all $t \geq T$, we have $a_{12}(t, u) \neq 0$,

$$
\begin{aligned}
& \Re[\xi(t)] \geq 0, \quad \xi(t) \equiv \frac{1}{2} \frac{d}{d t} \ln \left(1+2 \xi(T) \int_{T}^{t} e^{\int_{T}^{s} \int_{T}^{y} 2 R A(z, u) d z d y} d s\right), \\
& |\xi(t)|^{2}+\left|\operatorname{Tr}(A(t))+\frac{a_{12}^{\prime}(t, u)}{a_{12}(t, u)}-\int_{T}^{t} 2 R A(s, u) d s-2 a_{11}(t, u)\right|^{2} \\
& \quad \leq\left|a_{12}(t, u)\right|^{2}\left(c|\xi(t)|^{2 \alpha}-1\right),
\end{aligned}
$$

and (3.3) (or (3.6)) are satisfied with $J(t, u(t))=J_{8}(t)$, where

$$
J_{8}(t)=J_{0}(t)+\Re\left[\operatorname{Tr}(A(t))+\frac{a_{12}^{\prime}(t, u)}{a_{12}(t, u)}+(2 \alpha-1) \int_{T}^{t} 2 R A(s, u) d s+4(1-\alpha) \xi(t)\right] \text {, }
$$




$$
J_{0}(t)=\left|\int_{T}^{t} R A(s, u) d s\right|^{2}\left[\frac{1+\left|1+2 \xi(T) \int_{T}^{t} e^{\int_{T}^{s} \int_{T}^{y} 2 R A(z, u) d z d y}\right|^{2}}{2|\xi(T)| e^{\int_{T}^{t} \int_{T}^{s} \Re[2 R A(y, u)] d y d s}}\right] .
$$

Then the solution $u(t)$ of system (1.1) is stable (or asymptotically stable).

Example 4.1 From Theorem 4.3 it follows that system (1.1) with

$$
\begin{aligned}
& a_{11}=0, \quad a_{12}=1, \quad a_{21}=-t^{2 \beta}-\beta t^{\beta-1}-(\gamma+1)(\gamma+2) t^{\gamma}, \quad a_{22}=-2 t^{\beta}, \\
& -1<\beta \leq 0, \gamma \leq \frac{\beta}{2}-2
\end{aligned}
$$

(small damping) is asymptotically stable.

By using Jeffreys-Wentzel-Kramers-Brillouin (JWKB) approximation, we will prove the following theorem.

Theorem 4.4 Suppose that for a solution $u(t)$ of $(1.1) A(t, u) \in C^{4}(T, \infty)$, for all $t \geq T$, the conditions $a_{12}(t, u) \neq 0,(4.1)$,

$$
\begin{aligned}
& |R A(t)|+\left|\operatorname{Tr}(A(t))+\frac{a_{12}^{\prime}(t, u)}{a_{12}(t, u)}-\frac{R A^{\prime}(t)}{2 R A(t)}-2 a_{11}(t, u)\right|^{2} \\
& \quad \leq\left|a_{12}(t)\right|^{2}\left(c|R A(t)|^{\alpha}-1\right)
\end{aligned}
$$

and (3.3) (or (3.6)) are satisfied, where $J(t, u(t))=J_{9}(t)$,

$$
\begin{aligned}
& J_{9}[t]=J_{0}(t)+\Re\left[2 i \sqrt{R A(t)}+\frac{(2 \alpha-1) R A^{\prime}(t, u)}{2 R A(t)}+\operatorname{Tr}(A(t))+\frac{a_{12}^{\prime}(t, u)}{a_{12}(t, u)}\right], \\
& J_{0}(t)=\frac{1}{2}\left|R A^{-1 / 4}\left(R A^{-1 / 4}\right)^{\prime \prime}(t, u)\right|\left(\left|e_{2 i \sqrt{R A}}(t)\right|+\left|e_{-2 i \sqrt{R A}}(t)\right|\right) .
\end{aligned}
$$

Then the solution $u(t)$ of system (1.1) is stable (or asymptotically stable).

The following theorem is proved by using the Hartman-Wintner approximation [14].

Theorem 4.5 Suppose for a solution $u(t)$ of system $(1.1), A(t, u) \in C^{3}(T, \infty)$, there exist the constants $c>0, \alpha$ such that and for $t \geq T$, we have $a_{12}(t, u) \neq 0$,

$$
\begin{aligned}
& \Re[s] \geq 0, \quad s=i \sqrt{R A(t)\left(1-r^{2}(t)\right)}, \\
& r(t) \equiv \frac{R A^{\prime}(t, u)}{4 R A^{3 / 2}(t, u)}, \quad w(t) \equiv \frac{r^{\prime}(t) r(t)}{r^{2}(t)-1}, \\
& \left|\operatorname{Tr}(A(t))+\frac{a_{12}^{\prime}(t, u)}{a_{12}(t, u)}-\frac{R A^{\prime}(t)}{2 R A(t)}-2 a_{11}(t, u)\right|^{2}+\left|R A\left(r^{2}-1\right)\right| \\
& \quad \leq\left|a_{12}\right|^{2}\left(c\left|R A\left(r^{2}-1\right)\right|^{\alpha}-1\right)
\end{aligned}
$$

and (3.3) (or (3.6)) are satisfied, where $J(t, u(t))=J_{10}(t)$,

$$
\begin{aligned}
J_{10}(t)= & J_{0}(t)+\operatorname{Re}\left[2 \sqrt{R A\left(r^{2}-1\right)(t, u)}\right. \\
& \left.+\frac{(2 \alpha-1)\left[R A\left(r^{2}-1\right)\right]^{\prime}(t, u)}{2 R A\left(r^{2}-1\right)(t, u)}+\operatorname{Tr}(A(t))+\frac{a_{12}^{\prime}(t, u)}{a_{12}(t, u)}\right],
\end{aligned}
$$




$$
J_{0}(t)=\sqrt{(\operatorname{Re}[w(t)])^{2}+\frac{|w(t)|^{2}}{4}|| e_{-s}(t)|+| e_{s}(t)\left|+\sqrt{1-r^{-2}(t)}\left(\left|e_{-s}(t)\right|-\left|e_{s}(t)\right|\right)\right|^{2}} .
$$

Then the solution $u(t)$ of system (1.1) is stable (or asymptotically stable).

Remark 4.1 Note that if $R A(t) \geq 0$ and $r^{2}(t)<1$, then $\left|e_{s}(t)\right|=1$,

$$
J_{0}(t)=\sqrt{(\operatorname{Re}[w(t)])^{2}+|w(t)|^{2}} \leq|w(t)| \sqrt{2}, \quad w(t)=H A(t)=\frac{r^{\prime}(t) r(t)}{r^{2}(t)-1} .
$$

In this case, asymptotic stability condition (3.6) is simplified:

$$
\int_{T}^{\infty}\left(\frac{d}{d t} \ln \frac{\left|R A\left(1-r^{2}\right)\right|^{1 / 2-\alpha}(t, u)}{\left|a_{12}(t, u)\right|}-\operatorname{Tr}(A(t))-|w(t)| \sqrt{2}\right) d t=\infty
$$

Remark 4.2 For the Euler equation $u^{\prime \prime}(t)+R A(t) u(t)=0$ with $R A(t)=\frac{1}{4 t^{2}}$, we have $r(t) \equiv$ -1 , and the Hartman-Wintner approximation fails. To consider this case, one may consider the choice $\theta_{2}=-\frac{R A^{\prime}}{4 R A}=\frac{1}{2 t}$ with the other phase function $\theta_{1}=\frac{1}{2 t}+\frac{1}{t \ln (t)}$ that could be found by solving the equation $H A(t)=0$ (see (6.56)).

The following theorem is deduced from Theorem 4.1 by taking $p_{1}=\theta_{1}-\theta_{2}, p_{2}=0, \alpha=1$, $s=0$.

Theorem 4.6 Suppose that for a solution $u(t)$ of system $(1.1), A(t, u) \in C^{3}(T, \infty)$ and for $t \geq T$, we have $a_{12}(t, u) \neq 0$ and

$$
\begin{aligned}
& \Re[\sqrt{-R A(t)}] \geq 0, \\
& \left|\operatorname{Tr}(A(t))+\frac{a_{12}^{\prime}(t, u)}{a_{12}(t, u)}-2 a_{11}(t, u)\right|^{2}+|R A(t)| \leq\left|a_{12}\right|^{2}(c|R A(t)|-1), \\
& \int_{T}^{\infty} \Re\left[\frac{R A^{\prime}(t)}{2 R A(t)}+\operatorname{Tr}(A(t))+\frac{a_{12}^{\prime}(t, u)}{a_{12}(t, u)}+J_{0}(t)\right] d t=-\infty,
\end{aligned}
$$

where

$$
J_{0}(t)=\sqrt{4(\Re[\sqrt{-R A(t)}])^{2}+\left(\Re\left[\frac{R A^{\prime}(t)}{2 R A(t)}\right]\right)^{2}} .
$$

Then the solution $u(t)$ of system (1.1) is asymptotically stable.

\section{Stability theorems for the equations with real coefficients}

Theorem 5.1 Assume that for a solution $u_{1}(t)$ of $(2.1)$, the coefficients $P\left(t, u_{1}\right) \in C^{2}(T, \infty)$, $Q\left(t, u_{1}\right) \in C^{1}(T, \infty)$ are real-valued, for some positive constants $c_{j}, j=1,2$, the conditions

$$
\begin{aligned}
& R\left(t, u_{1}\right) \geq 0, \quad 1+\left|P\left(t, u_{1}\right)\right|^{2} \leq c_{2}\left|R\left(t, u_{1}\right)\right|, \quad t \geq T, \\
& \int_{T}^{\infty}\left(2 P\left(t, u_{1}\right)-\frac{\left|R^{\prime}\left(t, u_{1}\right)\right|+R^{\prime}\left(t, u_{1}\right)}{2 R\left(t, u_{1}\right)}\right) d t=\infty
\end{aligned}
$$


or

$$
\begin{aligned}
& R\left(t, u_{1}\right) \geq 0, \quad R\left(t, u_{1}\right)+\left|P\left(t, u_{1}\right)\right|^{2} \leq c_{3}, \\
& \int_{T}^{\infty}\left(2 P\left(t, u_{1}\right)-\frac{\left|R^{\prime}\left(t, u_{1}\right)\right|-R^{\prime}\left(t, u_{1}\right)}{2 R\left(t, u_{1}\right)}\right) d t=\infty
\end{aligned}
$$

are satisfied.

Then the solution $u_{1}(t)$ of equation (2.1) is asymptotically stable.

Example 5.1 By Theorem 5.1 the canonical linear equation

$$
v^{\prime \prime}(t)+2 b t^{\gamma-1} v^{\prime}(t)+c t^{2 \beta-2} v(t)=0, \quad b>0, c>0
$$

is asymptotically stable if one of the following conditions is satisfied:

(i) $0<\gamma<\beta$,

(ii) $\beta=\gamma>0, c-b^{2}>0$,

(iii) $\gamma=0, b>\beta-1>0$,

(iiii) $\gamma=0, b=1,0<\beta<2$.

A region of asymptotic stability of equation (5.3) described in Example 5.1 may be extended to

$$
0<\gamma \leq 2 \beta
$$

by using another asymptotic solution of (5.3) (see Example 5.4 or $[15,16])$.

Theorem 5.2 Assume that for a solution $u_{1}(t)$ of $(2.1)$, the coefficients $P\left(t, u_{1}\right) \in C^{2}(T, \infty)$, $Q\left(t, u_{1}\right) \in C^{1}(T, \infty)$ are real-valued, and for $t \geq T$,

$$
R\left(t, u_{1}\right) \geq 0, \quad \int_{T}^{\infty}\left(2 P\left(t, u_{1}\right)+\frac{R^{\prime}(t, u)}{2 R\left(t, u_{1}\right)}-\left|\frac{R^{\prime}\left(t, u_{1}\right)}{2 R\left(t, u_{1}\right)}\right|\right) d t=\infty
$$

Then the solution $u_{1}(t)$ of equation (2.1) approaches zero as $t \rightarrow \infty$.

Theorem 5.3 Assume that for a solution $u_{1}(t)$ of $(2.1)$, the coefficients $P\left(t, u_{1}\right) \in C^{1}(T, \infty)$, $Q\left(t, u_{1}\right) \in C(T, \infty)$ are real-valued, and for $t \geq T$,

$$
\begin{aligned}
& \left|P\left(t, u_{1}\right)\right| \leq C, \\
& \int_{T}^{\infty}\left(2 P\left(t, u_{1}\right)-\left|\frac{R\left(t, u_{1}(t)\right)}{k}-k\right|\right) d t=\infty \quad \text { for some positive number } k .
\end{aligned}
$$

Then the solution $u_{1}(t)$ of equation (2.1) is asymptotically stable.

Theorem 5.4 Suppose that for a solution $u_{1}(t)$ of $(2.1)$, the coefficients $P\left(t, u_{1}\right) \in C^{1}(T, \infty)$, $Q\left(t, u_{1}\right) \in C(T, \infty)$ are real functions, and condition (5.7) is satisfied. Then the solution $u_{1}(t)$ approaches zero as $t \rightarrow \infty$. 
Example 5.2 By Theorem 5.3 the equation

$$
v^{\prime \prime}(t)+2 b t^{\gamma-1} v^{\prime}(t)+\left(k^{2}+(\sigma+i \mu) t^{-\beta}\right) v(t)=0, \quad 1-\beta \leq \gamma \leq 1, b>\frac{1}{2 k}
$$

(where $\beta, \sigma, \mu$ are real numbers and $b, k, \gamma$ are positive numbers) is asymptotically stable.

Theorem 5.5 Assume that for a solution $u_{1}(t)$ of $(2.1)$, the coefficients $P\left(t, u_{1}\right) \in C^{1}(T, \infty)$, $Q\left(t, u_{1}\right) \in C(T, \infty)$ are real functions and

$$
\begin{aligned}
& \left|P\left(t, u_{1}\right)\right| \leq C, \quad t \geq T>0 \\
& \int_{T}^{\infty}\left(2 P\left(t, u_{1}\right)-\frac{2}{t}-\frac{2 t^{2}\left|R\left(t, u_{1}\right)\right|}{T}\right) d t=\infty, \quad t \geq T>0 .
\end{aligned}
$$

Then the solution $u_{1}(t)$ is asymptotically stable.

Theorem 5.6 Suppose that for a solution $u_{1}(t)$ of $(2.1)$, the coefficients $P\left(t, u_{1}\right) \in C^{1}(T, \infty)$, $Q\left(t, u_{1}\right) \in C^{2}(T, \infty)$ are real and condition (5.10) is satisfied. Then the solution $u_{1}(t)$ approaches zero as $t \rightarrow \infty$.

Example 5.3 By Theorem 5.5 the linear equation

$$
v^{\prime \prime}(t)+\frac{2 a v^{\prime}(t)}{t}+\left(\frac{a^{2}-a}{t^{2}}+\frac{b}{t^{3} \ln ^{2}(t)}\right) v(t)=0, \quad a>1
$$

is asymptotically stable.

Theorem 5.7 Assume that for a solution $u_{1}(t)$ of $(2.1)$, the coefficients $P\left(t, u_{1}\right) \in C^{3}(T, \infty)$, $Q\left(t, u_{1}\right) \in C^{2}(T, \infty)$ are real functions, and for all $t \geq T$,

$$
\begin{aligned}
& R\left(t, u_{1}\right)=Q\left(t, u_{1}\right)-P^{2}\left(t, u_{1}\right)-P^{\prime}\left(t, u_{1}(t)\right) \leq 0, \\
& 1+\left|P\left(t, u_{1}\right)+\frac{R^{\prime}\left(t, u_{1}\right)}{4 R\left(t, u_{1}\right)}\right|^{2} \leq c\left|R\left(t, u_{1}\right)\right|, \\
& \int_{T}^{\infty}\left(2 P-\frac{R^{\prime}}{2 R}-\left|\left((-R)^{-1 / 4}\right)^{\prime \prime}(-R)^{-1 / 4}-2 \sqrt{-R}\right|\right) d t=\infty .
\end{aligned}
$$

Then the solution $u_{1}(t)$ of $(2.1)$ is asymptotically stable.

Example 5.4 From Theorem 5.7 the asymptotic stability of the equation (see also $[9,15$, 16]) follows:

$$
v^{\prime \prime}(t)+2 b t^{\gamma-1} v^{\prime}(t)+c t^{2 \beta-2} v(t)=0, \quad b>0, c>0,1 \leq \beta<\gamma<2 \beta .
$$

Example 5.5 By Theorem 5.7, the nonlinear Matukuma equation

$$
u_{1}^{\prime \prime}+\frac{(n-1) u_{1}^{\prime}}{t}+\frac{A u_{1}\left|u_{1}\right|^{2 \beta}}{1+t^{2}}=0, \quad \beta>0, n>3
$$

is asymptotically stable. 
Theorem 5.8 Suppose that for a solution $u_{1}(t)$ of $(2.1)$, the coefficients $P\left(t, u_{1}\right) \in C^{1}(T, \infty)$, $Q\left(t, u_{1}\right) \in C^{2}(T, \infty)$ are real functions, and the conditions

$$
\begin{aligned}
& R\left(t, u_{1}(t)\right)\left(1-r^{2}(t)\right) \geq 0, \\
& \int_{T}^{\infty}\left(2 P\left(t, u_{1}\right)+\frac{R^{\prime}\left(t, u_{1}\right)}{2 R\left(t, u_{1}\right)}-4 \xi(t)-J_{0}\left(t, u_{1}\right)\right) d t=\infty
\end{aligned}
$$

are satisfied, where

$$
\begin{aligned}
& J_{0}(t)=\left|\left(\sqrt{r^{2}-1}-r\right)^{\prime}\right| \frac{1+\left|1+2 \xi_{0} \int_{T}^{t} \sqrt{\left|R\left(s, u_{1}\right)\right|} e^{2 i \int_{T}^{s} 2 \sqrt{R\left(1-r^{2}\right)(y)} d y} d s\right|^{2}}{2 \xi_{0}},
\end{aligned}
$$

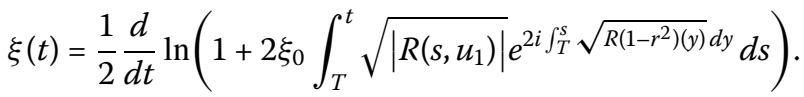

Then the solution $u_{1}(t)$ of (2.1) approaches zero as $t \rightarrow \infty$.

Remark 5.1 By taking $r(t)=\frac{R^{\prime}(t)}{4 R^{3 / 2}(t)} \in \mathrm{BV}(T, \infty), r(t) \leq \beta<1$, we get $J_{0}(t), \xi(t) \in L_{1}(T, \infty)$, and Theorem 5.8 becomes a version of Pucci-Serrin Theorem 1.3. In this case, (5.18) is simplified to

$$
\int_{T}^{\infty}\left(2 P(t)+\frac{R^{\prime}(t)}{2 R(t)}\right) d t=\lim _{t \rightarrow \infty}\left(\int_{T}^{t} 2 P(s) d s+\frac{1}{2} \ln \left|\frac{R(t)}{R(T)}\right|\right)=\infty
$$

Example 5.6 Due to Theorem 5.8, every solution of (1.6) with

$$
P(t)=0, \quad Q(t)=R(t)=\mu^{2} t^{2 \gamma} \ln ^{2 \sigma}(t), \quad \gamma>-1 \text { or } \gamma=-1, \sigma>0,
$$

approaches zero as $t \rightarrow \infty$, since

$$
r(t)=\frac{R^{\prime}}{4 R^{3 / 2}}=\frac{\gamma}{2 \mu t^{1+\gamma} \ln ^{1+\sigma}(t)}+\frac{\sigma}{2 \mu t^{1+\gamma} \ln ^{\sigma}(t)} \rightarrow 0, \quad t \rightarrow \infty
$$

Theorem 5.9 Suppose that for a solution $u_{1}(t)$ of $(2.1)$, the coefficients $P\left(t, u_{1}\right) \in C^{1}(T, \infty)$, $Q\left(t, u_{1}\right) \in C^{2}(T, \infty)$ are real functions, and for some constant $\xi_{0}>0$, we have

$$
\begin{aligned}
& \operatorname{Re}[\xi(t)] \geq 0, \quad \xi(t) \equiv \frac{1}{2} \frac{d}{d t} \ln \left(1+2 \xi_{0} \int_{T}^{t} \sqrt{|R(s) / R(T)|} e^{2 i} \int_{T}^{t} \sqrt{R(y) d y} d s\right), \\
& \int_{T}^{\infty} \Re\left(2 P\left(t, u_{1}\right)+\frac{R^{\prime}(t)}{2 R(t)}+2 i \sqrt{R(t)}-4 \xi(t)-J_{0}(t)\right) d t=\infty,
\end{aligned}
$$

where

$$
J_{0}(t)=\frac{\left|1+2 \xi_{0} \int_{T}^{t} \sqrt{|R(s) / R(T)|} e^{2 i \int_{T}^{s} 2 \sqrt{R(y) d y}} d s\right|^{2}+1}{2\left|R^{1 / 4}(t) \xi_{0} R^{-1 / 2}(T)\right|}\left|\left(R^{-1 / 4}\right)^{\prime \prime}(t)\right| .
$$

Then the solution $u_{1}(t)$ approaches zero as $t \rightarrow \infty$. 
Theorem 5.10 Suppose that for a solution $u_{1}(t)$ of $(2.1)$, the functions $P\left(t, u_{1}\right) \in C(T, \infty)$, $Q\left(t, u_{1}\right) \in C(T, \infty)$ are real and

$$
\begin{aligned}
& \int_{T}^{\infty} S\left(t, u_{1}\right)\left(1-2 S\left(t, u_{1}\right) \int_{T}^{\infty} e^{\int_{s}^{t}\left(2 P\left(y, u_{1}\right)-2 S\left(y, u_{1}\right)\right) d y} d s\right) d t=\infty, \\
& S\left(t, u_{1}\right) \equiv \int_{T}^{t} Q\left(s, u_{1}\right) e^{\int_{t}^{s} 2 P\left(y, u_{1}\right) d y} d s .
\end{aligned}
$$

Then the solution $u_{1}(t)$ of (2.1) approaches zero as $t \rightarrow \infty$.

If

$$
S^{2}\left(t, u_{1}\right) \int_{T}^{\infty} e^{\int_{s}^{t}\left(2 P\left(y, u_{1}\right)-2 S\left(y, u_{1}\right)\right) d y} d s \in L_{1}(T, \infty),
$$

then the attractivity condition (5.25) is simplified

$$
\int_{T}^{\infty} S\left(t, u_{1}\right) d t=\int_{T}^{\infty} \int_{T}^{t} Q\left(s, u_{1}\right) e^{-\int_{s}^{t} 2 P\left(y, u_{1}\right) d y} d s d t=\infty
$$

Note that (5.28) is Smith's [13] necessary and sufficient condition of asymptotic stability of (2.1) in the case of $Q(t)=1, P(t) \geq \varepsilon>0$.

Theorems 5.1-5.10 are new versions of the stability theorem proved in $[1-5,9-13,17-21]$ by a different technique of construction of the energy function.

\section{Proofs}

Lemma 6.1 Assume that all the solutions of linear system (1.1) are attractive to the origin, and functions $\chi_{1}, \chi_{2} \in C^{1}(T, \infty)$ are solutions of $C A\left(\chi_{j}\right)=0, j=1,2$. Then

$$
\lim _{t \rightarrow \infty} \frac{\left|a_{12}(t)\right| \exp \left\{\int_{T}^{t} \Re[\operatorname{Tr}(A(s))] d s\right\}}{\left|\chi_{1}(t)-\chi_{2}(t)\right|}=0 .
$$

Proof of Lemma 6.1 and Lemma 1.1 First, we derive formula (2.8) for the characteristic function. Solving for $u_{2}$ the first equation of (1.1), we get

$$
u_{2}(t)=\frac{u_{1}^{\prime}(t)-a_{11}(t, u(t)) u_{1}(t)}{a_{12}(t, u(t))} .
$$

To eliminate $u_{2}$, we substitute it in the second equation of (1.1) $u_{2}^{\prime}(t)=a_{21}(t, u(t)) u_{1}(t)+$ $a_{22}(t, u(t)) u_{2}(t)$, so we get (2.1): $L\left[u_{1}\right]=u_{1}^{\prime \prime}(t)+2 P u_{1}^{\prime}(t)+Q u_{1}(t)=0$, where $P, Q$ are as in (2.2). From definition (2.5), we get (2.8). Formula (A.22) (see the Appendix) for $C C A(t)$ is proved similarly by elimination of $u_{1}$.

The first component of a solution of linear system (1.1) may be represented in the Euler form

$$
u_{1}(t)=C_{1} e_{\chi_{1}}(t)+C_{2} e_{\chi_{2}}(t)
$$

where $\chi_{j}, j=1,2$ are solutions of $C A_{j}=0$. From $H A(t) \equiv 0$ we get

$$
\chi_{1}(t)+\chi_{2}(t)=\operatorname{Tr}(A(t))+\frac{a_{12}^{\prime}(t)}{a_{12}(t)}-\frac{\chi^{\prime}(t)}{\chi(t)}, \quad \chi(t) \equiv \frac{\chi_{1}(t)-\chi_{2}(t)}{2} .
$$


Since we are assuming that the solutions $e_{\chi_{j}}(t), j=1,2$ of linear system (1.1) are attractive to the origin, we have

$$
e^{\int_{T}^{t}\left(\chi_{1}+\chi_{2}\right) d s}=e^{\int_{T}^{t}\left(\operatorname{Tr}(A(s, u(s)))+a_{12}^{\prime}(s, u(s)) / a_{12}-\chi^{\prime}(s) / \chi\right) d s} \rightarrow 0
$$

as $t \rightarrow \infty$, that is, (6.1) is satisfied. Note that if additional condition (1.9) is satisfied, then (6.1) is also a sufficient condition of attractivity of solutions of (1.6), since in view of (6.5) as $t \rightarrow \infty$, we have

$$
\begin{aligned}
& \left|e^{\int_{T}^{t} \chi_{1} d s}\right|=e^{\int_{T}^{t} \Re\left[\chi_{1}-\chi_{2}\right] d s} e^{\int_{T}^{t} \Re\left[\chi_{1}+\chi_{2}\right] d s} \rightarrow 0, \\
& \left|e^{\int_{T}^{t} \chi_{2} d s}\right|=e^{\int_{T}^{t} \Re\left[\chi_{2}-\chi_{1}\right] d s} e^{\int_{T}^{t} \Re\left[\chi_{1}+\chi_{2}\right] d s} \rightarrow 0 .
\end{aligned}
$$

To prove Lemma 1.1, rewrite equation (1.6) in the form of system (1.1)

$$
\frac{d}{d t}\left(\begin{array}{c}
v(t) \\
v^{\prime}(t)
\end{array}\right)=\left(\begin{array}{cc}
0 & 1 \\
-Q(t) & -2 P(t)
\end{array}\right)\left(\begin{array}{c}
v(t) \\
v^{\prime}(t)
\end{array}\right)
$$

which means that

$$
\begin{aligned}
& a_{11}(t) \equiv 0, \quad a_{12}(t)=1, \\
& \operatorname{Tr}(A)=a_{22}(t)=-2 P(t), \quad \operatorname{det}(A)=-a_{21}(t)=Q(t) .
\end{aligned}
$$

Then (1.8) follows from (6.1).

Lemma 6.2 If $K(t)$ is a Hermitian $2 \times 2$ matrix with the entries $k_{i j}(t)$ such that

$$
\operatorname{det}(K(t)) \equiv k_{11}(t) k_{22}(t)-\left|k_{12}\right|^{2} \geq 0, \quad k_{22}(t)>0, t \geq T,
$$

then the matrix $K(t)$ is non-negative $(K(t) \geq 0)$, and for any 2-vector $u$

$$
u^{*} K(t) u \geq \frac{\operatorname{det}(K(t))}{\operatorname{Tr}(K(t))}|u|^{2}, \quad \operatorname{Tr}(K(t)) \equiv k_{11}(t)+k_{22}(t)
$$

\section{Remark 6.1 If}

$$
\operatorname{det}(K(t)) \equiv k_{11}(t) k_{22}(t)-\left|k_{12}\right|^{2} \geq 0, \quad k_{22}(t) \equiv 0, k_{11}(t) \geq 0, t \geq T,
$$

then $k_{12}(t) \equiv 0$, and

$$
u^{*} K(t) u=k_{11}\left|u_{1}\right|^{2} \geq 0
$$

Proof of Lemma 6.2 From the quadratic equation for the real eigenvalues of $K(t)$

$$
\lambda^{2}-\lambda \operatorname{Tr}(K(t))+\operatorname{det}(K(t))=0,
$$

we have

$$
\lambda_{1}=\frac{\operatorname{Tr}(K(t))+\sqrt{[\operatorname{Tr}(K(t))]^{2}-4 \operatorname{det}(K(t))}}{2}, \quad \lambda_{2}=\frac{\operatorname{det}(K(t))}{\lambda_{1}} .
$$


From $\operatorname{det}(K(t))=k_{11}(t) k_{22}(t)-\left|k_{12}(t)\right|^{2} \geq 0$, we have $k_{11}(t) \geq 0$ and

$$
\operatorname{Tr}(K(t))=k_{11}(t)+k_{22}(t)>0
$$

Further from

$$
0 \leq \lambda_{1} \leq \operatorname{Tr}(K(t)), \quad \lambda_{1} \geq \lambda_{2} \geq \frac{\operatorname{det}(K(t))}{\operatorname{Tr}(K(t))}
$$

we get

$$
u^{*} K(t) u \geq \lambda_{2}|u|^{2} \geq \frac{\operatorname{det}(K(t))}{\operatorname{Tr}(K(t))}|u|^{2} \geq 0
$$

Lemma 6.3 If there exist the complex-valued functions $p_{1}(t), p_{2}(t), \theta_{1,2} \in L_{1}(T, t)$, and a real-valued function $\beta(t) \in L_{1}(T, t)$ such that

$$
\begin{aligned}
& \beta(t)+2 \Re[H A(t)]+\frac{2 \Re\left[p_{1}(t)\left|e_{s}(t)\right|^{2}+p_{2}(t)\right]}{\left|e_{s}(t)\right|^{2}+1}>0, \\
& s(t)=\theta_{1}(t)-\theta_{2}(t)-p_{1}(t)+p_{2}(t), \\
& \beta(t) \geq J_{0}(t)-\Re\left[H A+p_{1}+p_{2}\right],
\end{aligned}
$$

where $J_{0}(t)$ is defined in (3.5), then the energy inequality

$$
V_{1}(t)+V_{2}(t) \leq C e_{\beta}(t)
$$

is satisfied, where the energy functions are defined in a more general form than in (2.14):

$$
V_{j}(t)=\frac{\left|\left(\theta_{j}(t)-a_{11}(t)\right) u_{1}(t)-a_{12}(t) u_{2}(t)\right|^{2}}{\left|\theta(t) e_{p_{j}+\theta_{3-j}}(t)\right|^{2}}, \quad j=1,2 .
$$

Proof of Lemma 6.3 Denoting

$$
Z_{j}=\left(\begin{array}{cc}
\left|d_{j}\right|^{2} & -\overline{d_{j}} a_{12}(t, u(t)) \\
-d_{j} \overline{a_{12}(t, u(t))} & \left|a_{12}(t, u(t))\right|^{2}
\end{array}\right), \quad d_{j}=\theta_{j}(t)-a_{11}(t, u(t)), \quad j=1,2,
$$

we can rewrite energy formula (6.20) in the form

$$
V_{j}(t)=\frac{u^{*} Z_{j} u}{\left|\theta e_{p_{j}+\theta_{3-j}}\right|^{2}}, \quad j=1,2, \quad V_{1}(t)=\frac{u^{*} Z_{1} u}{\left|\theta e_{p_{1}+\theta_{2}}\right|^{2}}=\frac{u^{*} Z_{1} u}{\left|\theta e_{p_{2}+\theta_{1}}\right|^{2}}\left|e_{s}\right|^{2} .
$$

By differentiation, we get

$$
\begin{aligned}
& V_{j}^{\prime}(t)=\frac{u^{*} Y_{j} u}{\left|\theta e_{p_{j}+\theta_{3-j}}\right|^{2}}, \\
& Y_{j}=Z_{j}^{\prime}+A^{*} Z_{j}+Z_{j} A-2 Z_{j} \Re\left[p_{j}+\theta_{3-j}+\theta^{\prime} / \theta\right], \quad j=1,2,
\end{aligned}
$$




$$
\begin{aligned}
& V_{1}(t)+V_{2}(t)=\frac{u^{\prime \prime}\left[Z_{1}\left|e_{s}(t)\right|^{2}+Z_{2}\right] u}{\left|\theta e_{p_{2}+\theta_{1}}(t)\right|^{2}}, \\
& \beta\left(V_{1}+V_{2}\right)-V_{1}^{\prime}-V_{2}^{\prime}=\frac{u^{*}\left[\left(\beta Z_{1}-Y_{1}\right)\left|e_{s}\right|^{2}+\beta Z_{2}-Y_{2}\right] u}{\left|\theta e_{p_{2}+\theta_{1}}(t)\right|^{2}}=\frac{u^{*} N u}{\left|\theta e_{p_{2}+\theta_{1}}(t)\right|^{2}},
\end{aligned}
$$

where

$$
N=\left(\beta Z_{1}-Y_{1}\right)\left|e_{s}(t)\right|^{2}+\beta Z_{2}-Y_{2}
$$

By direct calculations

$$
N=\left(\begin{array}{cc}
A_{1}+\overline{A_{1}}+\beta_{1} A_{0} & a_{12}\left(\overline{A_{2}}-\beta_{1} \overline{A_{3}}\right) \\
\overline{a_{12}}\left(A_{2}-\beta_{1} A_{3}\right) & \left|a_{12}\right|^{2}\left(A_{4}+\beta_{1} A_{5}\right)
\end{array}\right),
$$

where

$$
\begin{aligned}
& A_{0}=\left|d_{1} e_{s}\right|^{2}+\left|d_{2}\right|^{2}, \quad A_{1}=\left(p_{1}-\frac{C A_{1}}{d_{1}}\right)\left|d_{1} e_{s}\right|^{2}+\left(p_{2}-\frac{C A_{2}}{d_{2}}\right)\left|d_{2}\right|^{2}, \\
& A_{2}=\left(\frac{C A_{1}}{d_{1}}-p_{1}-\overline{p_{1}}\right) d_{1}\left|e_{s}\right|^{2}+C A_{2}-d_{2}\left(p_{2}+\overline{p_{2}}\right), \quad A_{3}=d_{1}\left|e_{s}\right|^{2}+d_{2}, \\
& A_{4}=\left(p_{1}+\overline{p_{1}}\right)\left|e_{s}\right|^{2}+p_{2}+\overline{p_{2}}, \quad A_{5}=1+\left|e_{s}\right|^{2}, \quad A_{0} A_{5}-\left|A_{3}\right|^{2}=\left|e_{s} \theta_{12}\right|^{2}, \\
& \beta_{1}=\beta+2 \operatorname{Re}[H A], \quad H A_{1}=H A-p_{1}-p_{2} .
\end{aligned}
$$

Further

$$
\operatorname{det}[N]=n_{11} n_{22}-n_{12} n_{21}=\left|a_{12} e_{s} \theta_{12}\right|^{2}\left[\beta_{1}^{2}-2 \beta_{1} \Re\left(H A_{1}\right)-F\right],
$$

where

$$
F=\frac{\left|A_{2}\right|^{2}-\left(A_{1}+\overline{A_{1}}\right) A_{4}}{\left|e_{s} \theta_{12}\right|^{2}}
$$

or

$$
\begin{aligned}
F= & \left|\frac{C A_{1}\left|e_{s}\right|+C A_{2}\left|e_{-s}\right|}{\theta_{1}-\theta_{2}}\right|^{2}-\left(p_{1}+\overline{p_{1}}\right)\left(\frac{C A_{2}}{\theta_{1}-\theta_{2}}+\frac{\overline{C A_{2}}}{\overline{\theta_{1}-\theta_{2}}}+p_{2}+\overline{p_{2}}\right) \\
& +\left(p_{2}+\overline{p_{2}}\right)\left(\frac{C A_{1}}{\theta_{1}-\theta_{2}}+\overline{\overline{C A_{1}}}\right),
\end{aligned}
$$

or

$$
\begin{aligned}
F(t)= & \left|\frac{C A_{1}(t)\left|e_{s}(t)\right|}{\theta_{1}(t)-\theta_{2}(t)}-\frac{\overline{C A_{2}(t)}\left|e_{-s}(t)\right|}{\overline{\theta_{1}(t)-\theta_{2}(t)}}\right|^{2} \\
& +\left[\Re\left(p_{2}(t)-p_{1}(t)+\frac{C A_{1}(t)+C A_{2}(t)}{\theta_{1}(t)-\theta_{2}(t)}\right)\right]^{2}-\left(\operatorname{Re}\left[H A(t)-p_{1}(t)-p_{2}(t)\right]\right)^{2},
\end{aligned}
$$

or using notation (3.5), we get

$$
F(t)=J_{0}^{2}(t)-\left[\Re\left(H A_{1}(t)\right)\right]^{2}, \quad J_{0}=\sqrt{F^{2}+\left(\Re\left[H A_{1}\right]\right)^{2}} .
$$


By Lemma 6.2 to have the non-negativity of the matrix $N$ (with the entries $n_{k j}$ ), it is sufficient to show that

$$
n_{22}=\left|a_{12}\right|^{2}\left[\beta_{1}\left(1+\left|e_{s}\right|^{2}\right)+2 \operatorname{Re}\left(p_{1}\left|e_{s}\right|^{2}+p_{2}\right)\right]>0, \quad \operatorname{det}[N] \geq 0 .
$$

The first condition is condition (6.17), and the second condition follows from (6.18) and (6.31):

$$
\beta_{1}=\beta+2 \Re[H A] \geq \Re\left[H A-p_{1}-p_{2}\right]+J_{0}=\Re\left[H A_{1}\right]+\sqrt{\left(\Re\left[H A_{1}\right]\right)^{2}+F} .
$$

So, from conditions (6.17), (6.18) it follows $N=\left(\beta Z_{1}-Y_{1}\right)\left|e_{s}(t)\right|^{2}+\beta Z_{2}-Y_{2} \geq 0$,

$$
V_{1}^{\prime}(t)+V_{2}^{\prime}(t)=\frac{u^{\prime \prime}\left(Y_{1}\left|e_{s}\right|^{2}+Y_{2}\right) u}{\left|\theta e_{\theta_{1}+p_{2}}\right|^{2}} \leq \beta \frac{u^{*}\left(Z_{1}\left|e_{s}\right|^{2}+Z_{2}\right) u}{\left|\theta e_{\theta_{1}+p_{2}}\right|^{2}}=\beta(t)\left(V_{1}+V_{2}\right)
$$

or (6.19) by integration.

Lemma 6.4 If the phase functions $\theta_{j}$ are such that (3.1) is satisfied, then

$$
V_{1}(t)+V_{2}(t) \geq \frac{c|u(t)|^{2}}{\left|\theta_{1}-\theta_{2}\right|^{2 \alpha}\left|e_{\theta_{1}+p_{2}}\right|^{2}}
$$

Proof of Lemma 6.4 Introducing the Hermitian matrix $K(t)$ with the entries $k_{i j}(t)$

$$
\begin{aligned}
K(t) & =Z_{1}\left|e_{s}(t)\right|^{2}+Z_{2} \\
& =\left(\begin{array}{cc}
\left|d_{1} e_{s}(t)\right|^{2}+\left|d_{2}\right|^{2} & -a_{12}\left(\overline{d_{1}\left|e_{s}(t)\right|^{2}+d_{2}}\right) \\
-\overline{a_{12}}\left(d_{1}\left|e_{s}(t)\right|^{2}+d_{2}\right) & \left|a_{12}\right|^{2}\left(1+\left|e_{s}(t)\right|^{2}\right),
\end{array}\right),
\end{aligned}
$$

we have

$$
\begin{aligned}
& \operatorname{det}(K(t))=\left|a_{12}\left(d_{1}-d_{2}\right) e_{s}(t)\right|^{2}=\left|a_{12}\left(\theta_{1}-\theta_{2}\right) e_{s}(t)\right|^{2}, \\
& \operatorname{Tr}(K(t))=\left|a_{12}\right|^{2}\left(1+\left|e_{s}(t)\right|^{2}\right)+\left|d_{1} e_{s}(t)\right|^{2}+\left|d_{2}\right|^{2} .
\end{aligned}
$$

From condition (3.1) we get

$$
\operatorname{Tr}(K(t)) \leq c\left|a_{12} e_{s}\left(\theta_{1}-\theta_{2}\right)^{\alpha}\right|^{2}, \quad \frac{\operatorname{Tr}(K(t))}{\operatorname{det}(K(t))} \leq c\left|\theta_{1}-\theta_{2}\right|^{2 \alpha-2}
$$

Further, by using Lemma 6.2, we obtain (6.35)

$$
V_{1}+V_{2}=\frac{u^{*} K u}{\left|\theta e_{\theta_{1}+p_{2}}\right|^{2}} \geq \frac{\operatorname{det}(K)|u|^{2}}{\operatorname{Tr}(K)\left|\theta e_{\theta_{1}+p_{2}}\right|^{2}} \geq \frac{c|u(t)|^{2}}{\left|\theta_{1}-\theta_{2}\right|^{2 \alpha}\left|e_{\theta_{1}+p_{2}}\right|^{2}}
$$

Proof of Theorem 3.1 First let us check that under the conditions of Theorem 3.1, Lemma 6.3 is applicable. Condition (6.18) is satisfied by choosing

$$
\beta(t)=J_{0}(t)-\Re\left[H A+p_{1}+p_{2}\right], \quad J_{0}(t)=\sqrt{F+\left[\Re\left(H A-p_{1}-p_{2}\right)\right]^{2}} .
$$


Condition (6.17) is satisfied as well in view of condition (3.2)

$$
\left(1+\left|e_{s}\right|^{2}\right) J_{0}(t)+\Re\left[\left|e_{s}\right|^{2}\left(H A_{1}+2 p_{1}\right)+H A_{1}+2 p_{2}\right]>0
$$

From Lemma 6.3 and Lemma 6.4, we get

$$
\begin{aligned}
& \frac{c|u|^{2}}{\left|\left(\theta_{1}-\theta_{2}\right)^{\alpha} e_{\theta_{1}+p_{2}}\right|^{2}} \leq V_{1}+V_{2} \leq C e_{\beta}(t), \quad e_{\beta}(t) \equiv e^{\int_{T}^{t} \beta(s) d s}, \\
& c|u(t)|^{2} \leq C e_{\beta+2 \Re\left(\theta_{1}+p_{2}+\alpha \theta^{\prime} \mid \theta\right)}=C e_{J_{1}}(t),
\end{aligned}
$$

where $J_{1}(t)$ is defined as in (3.13):

$$
J_{1}=\Re\left(2 \theta_{1}+2 \alpha \theta^{\prime} / \theta-H A+p_{2}-p_{1}\right)+J_{0}(t), \quad J_{0}(t)=\sqrt{F+\left[\Re\left(H A_{1}\right)\right]^{2}} .
$$

Substituting here formula (2.9) for $H A(t)$, we get (3.4). Further from (3.3) and (6.43) the boundedness of $|u(t)|$ and the stability follow.

Proof of Remark 3.2 Note that if for linear system (1.1) $\theta_{j}(t)=\chi_{j}(t), j=1,2, \Re\left[\chi_{1}(t)-\right.$ $\left.\chi_{2}(t)\right] \geq 0, \Re\left[p_{2}(t)-p_{1}(t)\right] \equiv 0$, then $C A_{1,2}(t) \equiv 0, J_{0}(t) \equiv 0$, and solutions of (1.1) could be represented in the form (see (6.2))

$$
\begin{aligned}
& u_{1}(t)=C_{1} e^{\int_{T}^{t} \chi_{1}(s) d s}+C_{2} e^{\int_{T}^{t} \chi_{2}(s) d s} \\
& u_{2}(t)=\frac{C_{1}\left(\chi_{1}(t)-a_{11}(t)\right) e^{\int_{T}^{t} \chi_{1}(s) d s}+C_{2}\left(\chi_{2}(t)-a_{11}(t)\right) e^{\int_{T}^{t} \chi_{2}(s) d s}}{a_{12}(t)} .
\end{aligned}
$$

Solution $u(t)=\left(u_{1}, u_{2}\right)$ of (1.1) is bounded and stable if and only if for all $t \geq T$ and $j=1,2$

$$
\exp \int_{T}^{t} \Re\left[\chi_{j}(s)\right] d s \leq \text { const }, \quad\left|\frac{\chi_{1}(t)-a_{11}(t)}{a_{12}(t)}\right| \exp \int_{T}^{t} \Re\left[\chi_{j}(s)\right] d s \leq \text { const }
$$

These exact conditions are close to conditions (3.1), (3.3) of Theorem 3.1 which, under assumption $\theta_{j}(t)=\chi_{j}(t), \mathfrak{R}\left[\chi_{1}(t)-\chi_{2}(t)\right] \geq 0$, turn to (see also (3.13))

$$
\begin{aligned}
& \left|\frac{\chi_{1}(t)-a_{11}(t)}{a_{12}(t)}\right|^{2}+\left|\frac{\chi_{1}(t)-a_{11}(t)}{a_{12}(t)} e_{-s}(t)\right|^{2}+2 \leq c\left|\chi_{1}(t)-\chi_{2}(t)\right|^{2 \alpha}, \\
& \int_{T}^{t}\left(\Re\left[\chi_{1}(s)\right]+\frac{\alpha\left(\chi_{1}^{\prime}(t)-\chi_{2}^{\prime}(t)\right)}{\chi_{1}(t)-\chi_{2}(t)}\right) d s \leq \text { const. }
\end{aligned}
$$

Proof of Theorem 3.2 From (3.1), (3.2) we get estimate (6.43) as in the proof of Theorem 3.1. Further from (3.6) and (6.43) the boundedness of $|u(t)|$ and $|u(t)|^{2} \rightarrow 0$ as $t \rightarrow \infty$, that is, the asymptotic stability, follow.

Proof of Theorem 3.3 Choosing

$$
\beta(t)=-J_{0}(t)-\Re\left[H A+p_{1}+p_{2}\right],
$$


we have again $\operatorname{det}(N) \geq 0$. In view of

$$
\beta_{1}=\beta+2 \Re[H A(t)]=\Re\left[H A(t)-p_{1}-p_{2}\right]-J_{0}(t)
$$

from assumption (3.7), we have $\operatorname{Re}[H A]<J_{0}-\operatorname{Re}\left[p_{1}-p_{2}\right]$ and $\operatorname{Re}[H A]<J_{0}+\operatorname{Re}\left[p_{1}-p_{2}\right]$, and

$$
\begin{aligned}
& \beta_{1}+p_{1}+\overline{p_{1}}<0, \quad \beta_{1}+p_{2}+\overline{p_{2}}<0, \\
& \frac{n_{22}}{\left|a_{12}\right|^{2}}=A_{4}+\beta_{1} A_{5}=\left|e_{s}\right|^{2}\left(\beta_{1}+p_{1}+\overline{p_{1}}\right)+\beta_{1}+p_{2}+\overline{p_{2}}<0,
\end{aligned}
$$

which implies $n_{22}<0$, $\operatorname{det}(N) \geq 0, N(t) \leq 0$, and from (6.25) $Y_{1}\left|e_{s}\right|^{2}+Y_{2} \geq \beta\left(\left(Z_{1}\left|e_{s}\right|^{2}+Z_{2}\right)\right)$.

So,

$$
V_{1}^{\prime}(t)+V_{2}^{\prime}(t)=\frac{u^{\prime \prime}\left(Y_{1}\left|e_{s}\right|^{2}+Y_{2}\right) u}{\left|\theta e_{\theta_{1}}\right|^{2}} \geq \beta \frac{u^{\prime \prime}\left(Z_{1}\left|e_{s}\right|^{2}+Z_{2}\right) u}{\left|\theta e_{\theta_{1}+p_{2}}\right|^{2}}=\beta(t)\left(V_{1}+V_{2}\right)
$$

or by integration

$$
\frac{\mu|u|^{2}}{\left|\theta e_{\theta_{1}+p_{2}}\right|^{2}} \geq \frac{u^{*} K u}{\left|\theta e_{\theta_{1}+p_{2}}\right|^{2}}=V_{1}(t)+V_{2}(t) \geq C e_{\beta},
$$

where $\mu$ is the largest eigenvalue of the non-negative matrix $K=Z_{1}\left|e_{s}\right|^{2}+Z_{2}$.

Since both eigenvalues of the matrix $K$ are non-negative, we have

$$
\begin{aligned}
& \mu \leq \operatorname{Tr}(K)=\left|d_{1} e_{s}\right|^{2}+\left|d_{2}\right|^{2}+\left|a_{12}\right|^{2}\left(1+\left|e_{s}\right|^{2}\right), \\
& |u(t)|^{2} \geq \frac{C e_{\operatorname{Re}\left[\beta+2 \theta_{1}+2 p_{2}+2 \theta^{\prime} \mid \theta\right]}(t)}{\mu} \geq \frac{C e_{J_{2}}(t)}{\left|d_{1} e_{s}\right|^{2}+\left|d_{2}\right|^{2}+\left|a_{12}\right|^{2}\left(1+\left|e_{s}\right|^{2}\right)} .
\end{aligned}
$$

From this estimate and (3.8), it follows $|u(t)|^{2} \rightarrow \infty$ as $t \rightarrow \infty$.

Proof of Example 3.1 We have

$$
P(t)=b t^{\gamma-1}, \quad R=Q-P^{2}-P^{\prime}=c t^{2 \beta-2}-b^{2} t^{2 \gamma-2}+b(1-\gamma) t^{\gamma-2} \geq 0
$$

for $t \geq T$, and $T$ sufficiently big positive. Choosing

$$
\theta_{1}=i \sqrt{R}-\frac{R^{\prime}}{4 R}-P(t), \quad \theta_{2}=-i \sqrt{R}-\frac{R^{\prime}}{4 R}-P(t), \quad p_{1}=p_{2}=0,
$$

we have

$$
\begin{aligned}
& \theta=i \sqrt{R}, \quad\left|e_{s}(t)\right|=\left|e_{2 \theta}\right|=1, \quad \frac{\theta^{\prime}}{\theta}=\frac{R^{\prime}}{2 R}, \\
& C L_{1}=C L_{2}=\frac{5 R^{\prime 2}}{16 R^{2}}-\frac{R^{\prime \prime}}{4 R}, \quad H L \equiv 0, \\
& J_{0}(t)=\left|\frac{C L_{1}+C L_{2}}{2 \theta}\right|=\left|\frac{5 R^{\prime 2}}{32 R^{5 / 2}}-\frac{R^{\prime \prime}}{32 R^{3 / 2}}\right|=O\left(t^{-1-\beta}\right) \in L_{1}(T, \infty),
\end{aligned}
$$




$$
\begin{aligned}
J_{2}(t) & =\Re\left[\theta_{1}-\theta_{2}+\frac{\theta^{\prime}}{\theta}-2 P\right]-J_{0}(t)=\frac{R^{\prime}(t)}{2 R(t)}-2 P(t)-J_{0}(t) \\
& =-2 b t^{\gamma-1}-O(1 / t) .
\end{aligned}
$$

So, conditions (3.7), (3.8) are satisfied, and from Theorem 3.3 it follows that equation (3.10) is unstable.

Proof of Theorem 3.4 Consider equation (2.1) written in the form

$$
\frac{d}{d t}\left(\begin{array}{l}
u_{1}(t) \\
u_{1}^{\prime}(t)
\end{array}\right)=\left(\begin{array}{cc}
0 & 1 \\
-Q\left(t, u_{1}\right) & -2 P\left(t, u_{1}\right)
\end{array}\right)\left(\begin{array}{l}
u_{1}(t) \\
u_{1}^{\prime}(t)
\end{array}\right) .
$$

Let us choose

$$
\beta(t)=J_{0}(t)-\Re\left[H A(t)+p_{1}(t)+p_{2}(t)\right], \quad H A(t) \equiv H L(t),
$$

where $J_{0}(t)$ is defined in (3.5) with $C A_{j}(t) \equiv C L_{j}(t)$. Then the conditions of Lemma 6.3 are satisfied, and we get from Lemma 6.3

$$
\frac{u^{*} K u}{\left|\theta e_{\theta_{1}+p_{2}}\right|^{2}}=V_{1}+V_{2} \leq C e_{\beta}(t)=C e_{J_{0}-\Re\left[H L+p_{1}+p_{2}\right]}(t),
$$

where the matrix $K$ is defined in (6.36). Since from (3.11) it follows $\left|e_{s}(t)\right| \geq 1$, by applying Lemma 6.2, we have

$$
u^{*} K u-\frac{\left|\left(\theta_{1}-\theta_{2}\right) e_{s}\right|^{2}}{1+\left|e_{s}\right|^{2}}\left|u_{1}\right|^{2} \geq 0
$$

or

$$
\left(\begin{array}{cc}
\left|d_{1} e_{s}\right|^{2}+\left|d_{2}\right|^{2}-\frac{\left|\left(\theta_{1}-\theta_{2}\right) e_{s}\right|^{2}}{1+\left|e_{s}\right|^{2}} & -\overline{d_{1}\left|e_{s}\right|^{2}-d_{2}} \\
-d_{1}\left|e_{s}\right|^{2}-d_{2} & 1+\left|e_{s}\right|^{2}
\end{array}\right) \geq 0 .
$$

It means that for equation (2.1) we get

$$
u^{\prime \prime} K u \geq \frac{\left|\left(\theta_{1}-\theta_{2}\right) e_{s}\right|^{2}\left|u_{1}\right|^{2}}{1+\left|e_{s}\right|^{2}}=\frac{\left|\left(\theta_{1}-\theta_{2}\right)\right|^{2}|v|^{2}}{1+\left|e_{-s}\right|^{2}}, \quad u_{1}=v, \quad u_{2}=v^{\prime}(t) .
$$

From (3.11) it follows $\left|e_{-s}(t)\right| \leq 1$, and we have also

$$
u^{*} K u \geq \frac{1}{2}\left|\left(\theta_{1}-\theta_{2}\right) v(t)\right|^{2} .
$$

Further, using notation (3.18), (2.6) from (6.52), (6.53), we get

$$
\frac{1}{2}|v(t)|^{2} \leq C e_{\operatorname{Re}\left[J_{0}-H L+2 \theta_{1}+p_{2}-p_{1}\right]}=C e_{\operatorname{Re}\left[J_{0}+\theta_{1}-\theta_{2}-\theta^{\prime} \mid \theta-2 P+p_{2}-p_{1}\right]}=C e_{J_{3}}(t),
$$

and from (3.6) it follows $v(t) \rightarrow 0, t \rightarrow \infty$.

Proof of Theorem 3.7 By substitution

$$
\xi_{j}(t)=\theta_{j}(t)-\frac{\operatorname{Tr} A(t)}{2}-\frac{a_{12}^{\prime}(t)}{2 a_{12}(t)}, \quad 2 \xi(t) \equiv \xi_{1}(t)-\xi_{2}(t) \equiv \theta_{1}(t)-\theta_{2}(t),
$$


functions (2.8), (2.9) may be simplified

$$
C A_{j}(t)=\xi_{j}^{\prime}(t)+\xi_{j}^{2}(t)+R A(t), \quad H A(t)=\xi_{1}(t)+\xi_{2}(t)+\frac{\xi^{\prime}(t)}{\xi(t)} .
$$

Theorem 3.7 follows from Theorem 3.1, Theorem 3.2 by taking a given function $\xi_{2}(t)$ and choosing $p_{1} \equiv p_{2} \equiv 0$, and phase function $\xi_{1}(t)$ as follows (see (6.55)):

$$
\begin{aligned}
& \xi_{1}(t)=\xi_{2}(t)+2 \xi(t), \quad \xi(t)=\frac{\xi(T) e^{-\int_{T}^{t} 2 \xi_{2} d y}}{1+2 \xi(T) \int_{T}^{t} e^{-\int_{T}^{s} 2 \xi_{2} d y} d s}, \\
& \frac{\xi^{\prime}(t)}{\xi(t)}=-2 \xi_{2}(t)-2 \xi(t), \quad H A(t)=\xi_{1}+\xi_{2}(t)+\frac{\xi^{\prime}(t)}{\xi(t)}=0, \\
& e_{s}(t)=e_{2 \xi}(t)=e^{\int_{T}^{t} 2 \xi(s) d s}=\frac{\xi(T)}{\xi(t)} e^{-\int_{T}^{t} 2 \xi_{2}(y) d s}=1+2 \xi(T) \int_{T}^{t} e^{-\int_{T}^{s} \xi_{2} d z} d s .
\end{aligned}
$$

Further from (6.56), (3.15)

$$
\begin{aligned}
& C A_{1}(t)=C A_{2}(t)=\xi_{2}^{\prime}(t)+\xi_{2}^{2}(t)+R A(t), \\
& J_{0}(t)=\frac{\left(1+\left|e_{2 \xi}\right|^{2}\right)\left|C A_{2}\right|}{2\left|\xi(t) e_{2 \xi}\right|}=\frac{1+\left|1+2 \xi(T) \int_{T}^{t} e^{-\int_{T}^{s} 2 \xi_{2} d y} d s\right|^{2}}{2\left|\xi(T) e^{-\int_{T}^{t} \Re\left[2 \xi_{2}\right] d s}\right|}\left|C A_{2}\right| .
\end{aligned}
$$

So, conditions (3.1), (3.2) turn to (3.29), (3.30). From (3.13) we have

$$
\begin{aligned}
J_{6}(t) & =J_{1}=J_{0}(t)+\Re\left[2 \theta_{1}+\frac{2 \alpha \xi^{\prime}}{\xi}\right] \\
& =J_{0}(t)+\Re\left[2 \xi_{2}+\operatorname{Tr}(A)+\frac{a_{12}^{\prime}}{a_{12}}+4 \xi(t)+\frac{2 \alpha \xi^{\prime}}{\xi}\right], \\
J_{6}(t) & =J_{0}(t)+\Re\left[\operatorname{Tr}(A)+\frac{a_{12}^{\prime}}{a_{12}}-2 \xi_{2}+\frac{2(\alpha-1) \xi^{\prime}}{\xi}\right]
\end{aligned}
$$

or (3.31).

Proof of Theorem 3.8 Theorem 3.8 follows from Theorem 3.4 applied to the system (6.51). By choosing $p_{1}=p_{2}=0$ and $\theta$ as in (3.31), in view of $H A=\xi_{1}+\xi_{2}+\frac{\xi_{1}^{\prime}-\xi_{2}^{\prime}}{\xi_{1}-\xi_{2}}=0$, we get (3.33) from (3.6) and (3.18).

Proof of Theorem 4.1 Theorem 4.1 follows from Theorems 3.1 and 3.2 by choosing, as the approximate solutions of $C A_{j}(t)=0$ (see (6.56)), the eigenvalue approximation

$$
\begin{aligned}
& \xi_{1,2}(t)= \pm \sqrt{-R A(t)}, \\
& z=\frac{C A_{1}}{\xi_{1}-\xi_{2}}=\frac{\xi_{1}^{\prime}+\xi_{1}^{2}+R A}{\xi_{1}-\xi_{2}}=\frac{R A^{\prime}}{4 R A}, \quad C A_{2}=-C A_{1}, \quad H A=\frac{R A^{\prime}}{2 R A}, \\
& s=\xi_{1}-\xi_{2}+p_{2}-p_{1}=2 \sqrt{-R A(t)}+p_{2}-p_{1} .
\end{aligned}
$$

Condition (3.1) turns to (3.12) (see Remark 3.3), or to (4.2). 
In view of (6.64) and

$$
|z| e_{s}|+\bar{z}| e_{-s}||^{2}=[\operatorname{Re}(2 z)]^{2}+|z|^{2}\left(\left|e_{s}\right|-\left|e_{-s}\right|\right)^{2}
$$

we get from (3.4), (3.5) formulas (4.3), (4.4):

$$
\begin{aligned}
J_{0} & =\sqrt{|z| e_{s}|+\bar{z}| e_{-s}||^{2}+\left(\operatorname{Re}\left[p_{1}-p_{2}\right]\right)^{2}} \\
& =\sqrt{[\operatorname{Re}(2 z)]^{2}+|z|^{2}\left(\left|e_{s}\right|-\left|e_{-s}\right|\right)^{2}+\left(\operatorname{Re}\left[p_{1}-p_{2}\right]\right)^{2}} .
\end{aligned}
$$

From (4.1) we have $\left|e_{s}(t)\right| \geq 1$, and condition (3.2) is satisfied since from (4.4) we have $\operatorname{Re}\left[J_{0}+H A\right]>0$.

Proof of Theorem 4.2 Theorem 4.2 follows from Theorems 3.1 and 3.2 by choosing $p_{2}(t)=$ $p_{1}(t)=0$, and the special Riccati equation approximation

$$
\xi_{1}=\frac{1}{t}, \quad \xi_{2}=0, \quad \theta_{1}-\theta_{2}=\xi_{1}-\xi_{2}=\frac{1}{t}, \quad T>0
$$

By direct calculations,

$$
\begin{aligned}
& C A_{1}(t)=\xi_{1}^{\prime}+\xi_{1}^{2}+R A=R A(t)=C A_{2}(t), \quad s=2 \xi=\xi_{1}-\xi_{2}=\frac{1}{t}, \\
& H A(t)=0, \quad e_{2 \xi}(t)=\frac{t}{T} \geq 1 .
\end{aligned}
$$

Condition (3.2) is true, since (3.11) is satisfied (see Remark 3.3). Condition (3.1) with $\alpha=0$ turns to (3.12): $2\left|\xi_{1}-\xi_{2}\right|^{2}+3\left|\theta_{1}-a_{11}\right|^{2}+2\left|a_{12}\right|^{2} \leq c\left|a_{12}\right|^{2}$ or

$$
\frac{2}{t^{2}}+3\left|\frac{1}{t}+\frac{\operatorname{Tr}(A)}{2}+\frac{a_{12}^{\prime}(t)}{2 a_{12}}-a_{11}\right|^{2} \leq c\left|a_{12}\right|^{2}
$$

which follows from (4.5). From (3.15), since $0<T \leq t$, we get

$$
J_{0}(t)=\left|\frac{C A_{1}\left|e_{2 \xi}\right|+C A_{2}\left|e_{-2 \xi}\right|}{\xi_{1}-\xi_{2}}\right|=t\left(\frac{t}{T}+\frac{T}{t}\right)|R A(t)| \leq \frac{2 t^{2}}{T}|R A(t)| .
$$

Further from (3.13) we get

$$
J_{1}=J_{0}+\Re\left[2 \xi_{1}-H A\right] \leq \frac{2 t^{2}}{T}|R A(t)|+\Re\left[\frac{2}{t}+\operatorname{Tr}(A)+\frac{a_{12}^{\prime}}{a_{12}}\right],
$$

and condition (3.6) turns to (4.6).

Proof of Theorem 4.3 Theorem 4.3 follows from Theorem 3.7 by choosing the linear equation approximation

$$
\xi_{2}=-\int_{T}^{t} R A(s) d s, \quad \xi_{2}^{\prime}+\xi_{2}^{2}+R A=\left(\int_{T}^{t} R A(s) d s\right)^{2} .
$$


Proof of Example 4.1 Example 4.1 follows from Theorem 4.3. Since $\gamma \leq-2$, we have

$$
\begin{aligned}
& R A(t)=\frac{a_{22}^{\prime}}{2}-\frac{a_{22}^{2}}{4}-a_{21}=(\gamma+1)(\gamma+2) t^{\gamma}, \quad \operatorname{Tr}(A)=-2 t^{\beta}, \\
& e^{\int_{T}^{t} \int_{T}^{y} R A(y) d y d s}=C \exp \left(t^{\gamma+2}\right) \rightarrow C e^{0}=C, \quad t \rightarrow \infty, \\
& \int_{T}^{t} e^{\int_{T}^{s} \int_{T}^{y} 2 R A(z) d z d y} d s=O(t), \quad t \rightarrow \infty .
\end{aligned}
$$

Choosing $\xi(T)=1$, by using l'Hospital's rule, if $\beta \leq 0, \gamma \leq-2<-1$, we get

$$
\begin{aligned}
& \frac{1+|1+| 2 \xi(T)\left|\int_{T}^{t} e^{\int_{T}^{s} \int_{T}^{y} \Re[2 R A(z)] d z d y}\right|^{2}}{2|\xi(T)| e^{\int_{T}^{t} \int_{T}^{s} \Re[2 R A(y)] d y d s}}=O\left(t^{2}\right), \quad \xi(t)=O\left(t^{-1}\right), \quad t \rightarrow \infty \\
& |\xi|^{2}+\left|2 t^{\beta}+(\gamma+2) t^{\gamma+1}\right|+1 \leq C
\end{aligned}
$$

and conditions (4.7), (4.8) with $\alpha=0$ are satisfied.

$$
\begin{aligned}
J_{0}(t) & =O\left(t^{2 \gamma+4}\right), \quad t \rightarrow \infty \\
J_{8}(t) & =J_{0}(t)+\Re\left[\operatorname{Tr} A+\frac{a_{12}^{\prime}}{a_{12}}\right]-\int_{T}^{t} 2 R A(s) d s+4 \xi \\
& =O\left(t^{2 \gamma+4}\right)-2 t^{\beta}-2(\gamma+2) t^{\gamma+1}+O\left(\frac{1}{t}\right) .
\end{aligned}
$$

Asymptotic stability condition (3.6) is satisfied as well:

$$
\int_{T}^{t} J_{8}(s) d s=O(\ln (t))-\frac{2 t^{\beta+1}}{\beta+1}-2 t^{\gamma+2}+O\left(\frac{t^{2 \gamma+5}}{2 \gamma+5}\right)+C \rightarrow-\infty, \quad t \rightarrow \infty
$$

Proof of Theorem 4.4 Theorem 4.4 follows from Theorem 3.1, Theorem 3.2 by choosing $p_{1}=p_{2}=0$, and JWKB approximation:

$$
\xi_{1,2}= \pm i \sqrt{R A(t)}-\frac{R A^{\prime}(t)}{4 R A(t)}, \quad \theta_{1}-\theta_{2}=\xi_{1}-\xi_{2}
$$

We have from (6.56), (3.15)

$$
\begin{aligned}
& s=\xi_{1}-\xi_{2}=2 i \sqrt{R A(t)}, \quad \frac{\xi_{1}^{\prime}-\xi_{2}^{\prime}}{\xi_{1}-\xi_{2}}=\frac{R A^{\prime}(t)}{2 R A(t)}, \quad H A=\xi_{1}+\xi_{2}+\frac{\xi_{1}^{\prime}-\xi_{2}^{\prime}}{\xi_{1}-\xi_{2}}=0, \\
& C A_{1}=C A_{2}=\xi_{1}^{\prime}+\xi_{1}^{2}+R A=\frac{5\left(R A^{\prime}\right)^{2}}{16 R A^{2}(t)}-\frac{R A^{\prime \prime}(t)}{4 R A(t)}=\left(R^{-1 / 4}\right)^{\prime \prime} R^{1 / 4}, \\
& J_{0}(t)=\frac{\left|C A_{1}\right| e_{s}|+| e_{-s}\left|C A_{2}\right|}{2|\xi(t)|}=\frac{1}{2}\left|\left(R A^{-1 / 4}\right)^{\prime \prime} R A^{-1 / 4}\right|\left(\left|e_{s}(t)\right|+\left|e_{-s}(t)\right|\right) .
\end{aligned}
$$

Conditions (3.11) and (3.2) are satisfied. Condition (3.1) turns to (3.12) or (4.11), and from (3.13) we get (4.12)

$$
J_{9}[t]=J_{1}(t)=J_{0}(t)+\Re\left[2 i \sqrt{R A(t)}+\frac{(2 \alpha-1) R A^{\prime}(t)}{2 R A^{\prime}(t)}+\operatorname{Tr}(A)+\frac{a_{12}^{\prime}}{a_{12}}\right] .
$$


Proof of Theorem 4.5 We deduce Theorem 4.5 from Theorems 3.1 and 3.2 assuming $p_{1}=$ $p_{2}=0$, and by choosing the Hartman-Wintner approximation [14]

$$
\xi_{j}(t)=q_{j}(t) \sqrt{R A(t)}, \quad j=1,2,
$$

where $q_{1,2}(t)$ are solutions of the quadratic equation $q_{j}^{2}+2 r q_{j}+1=0$,

$$
q_{1,2}(t)= \pm \sqrt{r^{2}(t)-1}-r(t), \quad r(t)=\frac{R A^{\prime}(t)}{4 R A^{3 / 2}}=-\frac{1}{2}\left(R A^{-1 / 2}\right)^{\prime}(t) .
$$

By calculations,

$$
\begin{array}{ll}
s=\xi_{1}-\xi_{2}=\left(q_{1}-q_{2}\right) \sqrt{R A}=2 \sqrt{R A\left(r^{2}-1\right)}, \quad \frac{\xi_{1}^{\prime}-\xi_{2}^{\prime}}{\xi_{1}-\xi_{2}}=\frac{\left[R A\left(r^{2}-1\right)\right]^{\prime}}{2 R A\left(r^{2}-1\right)}, \\
C A_{j}=q_{j}^{\prime} \sqrt{R A}+R A\left[q_{j}^{2}+2 r q_{j}+1\right]=q_{j}^{\prime} \sqrt{R A}, \quad z_{j}=\frac{C A_{j}}{\xi_{1}-\xi_{2}}=\frac{q_{j}^{\prime}}{\sqrt{r^{2}-1}} .
\end{array}
$$

Denoting

$$
\begin{aligned}
& z_{1}=\frac{\left(\sqrt{r^{2}-1}-r\right)^{\prime}}{2 \sqrt{r^{2}-1}}, \quad z_{2}=\frac{\left(-\sqrt{r^{2}-1}-r\right)^{\prime}}{2 \sqrt{r^{2}-1}}, \\
& w(t)=H A(t)=z_{1}-z_{2}=\frac{\left(r^{2}-1\right)^{\prime}}{2\left(r^{2}-1\right)}=\frac{r^{\prime}(t) r(t)}{r^{2}(t)-1},
\end{aligned}
$$

we have

$$
z_{1}=-\frac{w}{2}\left(\sqrt{1-r^{-2}}-1\right), \quad z_{2}=-\frac{w}{2}\left(\sqrt{1-r^{-2}}+1\right),
$$

and

$$
\left|z_{1}\right| e_{s}\left|+z_{2}\right| e_{-s}||^{2}=\frac{|w|^{2}}{4}\left|\left(\sqrt{1-r^{-2}}-1\right)\right| e_{s}|-| e_{-s}\left|\left(\sqrt{1-r^{-2}}+1\right)\right|^{2} .
$$

From (3.15)

$$
J_{0}(t)=\sqrt{(\operatorname{Re}[w])^{2}+\frac{|w|^{2}}{4}\left|\left(\sqrt{1-r^{-2}}-1\right)\right| e_{s}|-| e_{-s}\left|\left(\sqrt{1-r^{-2}}+1\right)\right|^{2}}
$$

or $(4.17)$

$$
J_{0}(t)=\sqrt{(\operatorname{Re}[w])^{2}+\frac{|w|^{2}}{4}|| e_{-s}|+| e_{s}\left|+\sqrt{1-r^{-2}}\left(\left|e_{-s}\right|-\left|e_{s}\right|\right)\right|^{2}},
$$

since $1-r^{-2} \leq 1,\left|e_{-s}\right| \leq 1$, we have

$$
J_{0}(t) \leq \sqrt{(\operatorname{Re}[w])^{2}+|w|^{2}} \leq|w| \sqrt{2} .
$$

From (3.4)

$$
J_{1}(t)=\operatorname{Re}\left[\xi_{1}-\xi_{2}+\frac{(2 \alpha-1)\left(\xi_{1}^{\prime}-\xi_{2}^{\prime}\right)}{\xi_{1}-\xi_{2}}+\operatorname{Tr}(A)+\frac{a_{12}^{\prime}}{a_{12}}\right]+J_{0}(t),
$$




$$
J_{1}(t)=\operatorname{Re}\left[2 \sqrt{R A\left(r^{2}-1\right)}+\frac{(2 \alpha-1)\left[R A\left(r^{2}-1\right)\right]^{\prime}}{2 R A\left(r^{2}-1\right)}+\operatorname{Tr}(A)+\frac{a_{12}^{\prime}}{a_{12}}\right]+J_{0}(t)
$$

From (4.14) we get $\left|e_{s}\right| \geq 1$, and in view of (3.12), condition (3.1) turns to

$$
\begin{aligned}
& \frac{3}{4}\left|2 \sqrt{\left(r^{2}-1\right) R A}-\frac{R A^{\prime}}{2 R A}+\operatorname{Tr}(A)+\frac{a_{12}^{\prime}}{a_{12}}-2 a_{11}\right|^{2}+8\left|R A\left(r^{2}-1\right)\right|+2\left|a_{12}\right|^{2} \\
& \quad \leq c\left|a_{12}\right|^{2}\left|R A\left(r^{2}-1\right)\right|^{\alpha},
\end{aligned}
$$

and it follows from (4.15).

From (4.17) we have $w=H A, \operatorname{Re}\left[H A+J_{0}\right]>0$, and condition (3.2) is satisfied.

To prove Remark 4.2, note that if $R A=\frac{1}{4 t^{2}}$, we have $r=-1$, and from the quadratic equation $q_{j}^{2}-2 r q_{j}+1=(q-1)^{2}=0$, we get $q=1$, or $\xi_{2}=-\frac{R A^{\prime}}{4 R A}=\frac{1}{2 t}$. Further, from the equation $H A=\frac{\xi^{\prime}}{\xi}+2 \xi+2 \xi_{2}=0$, we get $\xi=\frac{1}{2 t \ln (t)}$ and the other phase function $\xi_{1}=\xi_{2}+2 \xi=$ $\frac{1}{2 t}+\frac{1}{t \ln (t)}$.

Proof of Theorems 5.1, 5.2 Theorem 5.1 follows from Theorem 4.1 applied to system (6.51). Indeed, by substitution $R A(t) \rightarrow R(t), a_{12} \equiv 1, a_{11} \equiv 0, \operatorname{Tr}(A)=-2 P, p_{1}=p_{2}=0$, condition (4.2) of Theorem 4.1 turns to $|R|+|2 P|^{2}+1 \leq c|R|^{\alpha}$. Further, from condition $R \geq 0$, we get $\operatorname{Re}[s]=0$ and (4.1) is satisfied. From (4.4) we get $J_{0}=\left|\frac{R^{\prime}}{2 R}\right|$.

By choosing $\alpha=1$, the conditions of Theorem 4.1 turn to (5.1) (big damping case).

By choosing $\alpha=0$, the conditions of Theorem 4.1 turn to (5.2) (small damping case).

Theorem 5.2 follows from Theorem 3.4 by choosing

$$
\xi_{1,2}= \pm i \sqrt{R(t)}, \quad p_{1}=p_{2} \equiv 0
$$

Proof of Example 5.1 Since

$$
\begin{aligned}
& P(t)=b t^{\gamma-1}, \quad Q=c t^{2 \beta-2}, \\
& R=Q-P^{2}-P^{\prime}=c t^{2 \beta-2}-b^{2} t^{2 \gamma-2}+b(1-\gamma) t^{\gamma-2},
\end{aligned}
$$

from $\beta>\gamma>0$ we get $R(t)=Q(t)(1+o(1)) \geq 0$, and

$$
\frac{R^{\prime}(t)}{R(t)}-\frac{2(\beta-1)}{t}=t^{2 \gamma-2 \beta-1} O(1) \in L_{1}(T, \infty), \quad t \rightarrow \infty
$$

If $\beta \geq 1, \beta>\gamma>\gamma / 2>0$, then $c R(t) \geq 1+|P(t)|^{2}$, and condition (5.1) of Theorem 5.1 is satisfied:

$$
\int_{T}^{t}\left(2 P(t)-\frac{\left|R^{\prime}(t)\right|+R^{\prime}(t)}{2 R(t)}\right) d t=\int_{T}^{t}\left(2 b t^{\gamma-1}-\frac{(|\beta-1|+\beta-1)}{t}\right) d t+C \rightarrow \infty .
$$

If $0<\gamma<\beta \leq 1$ (small damping), then $|R| \leq C,|P| \leq C$, and condition (5.2) of Theorem 5.1 is satisfied:

$$
\int_{T}^{t}\left(2 P(t)-\frac{\left|R^{\prime}(t)\right|-R^{\prime}(t)}{2 R(t)}\right) d t=\int_{T}^{t}\left(2 b t^{\gamma-1}-\frac{(|\beta-1|-\beta+1)}{t}\right) d t+C \rightarrow \infty .
$$


If $\beta=\gamma \geq 1, c-b^{2}>0$, then condition (5.1) is satisfied again:

$$
\begin{aligned}
& R=\left(c-b^{2}\right) t^{2 \gamma-2}+b(1-\gamma) t^{\gamma-2}>0, \quad 1+|P|^{2} \leq C|R|, \\
& \frac{R^{\prime}(t)}{R(t)}-\frac{2(\gamma-1)}{t}=\frac{O(1)}{t^{1+\gamma}} \in L_{1}(T, \infty) .
\end{aligned}
$$

If $0<\beta=\gamma \leq 1, c-b^{2}>0$, then condition (5.2) is satisfied:

$$
R=\left(c-b^{2}\right) t^{2 \gamma-2}+b(1-\gamma) t^{\gamma-2} \geq 0, \quad|R| \leq C, \quad|P| \leq C .
$$

Further, if $\gamma=0, \beta \geq 1, b>\beta-1$, then in view of (6.87) condition (5.1) is satisfied:

$$
\begin{aligned}
& R(t)=c t^{2 \beta-2}+\left(b-b^{2}\right) t^{-2}>0, \quad 1+|P|^{2} \leq C R(t), \\
& \int_{T}^{t}\left(2 P(t)-\frac{\left|R^{\prime}(t)\right|+R^{\prime}(t)}{2 R(t)}\right) d t=\int_{T}^{t}\left(\frac{2 b-2(\beta-1)}{t}\right) d t \rightarrow \infty .
\end{aligned}
$$

If $\gamma=0,0<\beta<1, b>1-\beta$, then in view of (6.86) condition (5.2) is satisfied:

$$
\begin{aligned}
& |R|=\left|c t^{2 \beta-2}+\left(b-b^{2}\right) t^{-2}\right| \leq C, \quad|P|=C t^{-1} \leq C, \quad R^{\prime}(t) \leq 0, \\
& \int_{T}^{t}\left(2 P(t)+\frac{R^{\prime}(t)-\left|R^{\prime}(t)\right|}{2 R(t)}\right) d t=\int_{T}^{t}\left(2 P(t)+\frac{R^{\prime}(t)}{R(t)}\right) d t=\int_{T}^{t}\left(\frac{2 b+2 \beta-2}{t}\right) d t \rightarrow \infty .
\end{aligned}
$$

Finally, when $\gamma=0, b=1$, we have $P=\frac{1}{t}, P^{\prime}+P^{2}=0$,

$$
R=Q-P^{2}-P^{\prime}=Q=c t^{2 \beta-2}, \quad \frac{R^{\prime}}{2 R}=\frac{\beta-1}{t} .
$$

If $1 \leq \beta<2$, then $R^{\prime} \geq 0,1+|P|^{2} \leq C|R|$, and condition (5.1) is satisfied:

$$
\int_{T}^{\infty}\left(2 P(t)-\frac{\left|R^{\prime}(t)\right|+R^{\prime}(t)}{2 R(t)}\right) d t=\int_{T}^{\infty}\left(2 P(t)-\frac{R^{\prime}(t)}{R(t)}\right) d t=\int_{T}^{\infty} \frac{2+2-2 \beta}{t} d t=\infty .
$$

If $0<\beta \leq 1$, then $|P| \leq C,|R| \leq C, R^{\prime} \leq 0$, condition (5.2) is satisfied:

$$
\int_{T}^{\infty}\left(2 P(t)-\frac{\left|R^{\prime}(t)\right|-R^{\prime}(t)}{2 R(t)}\right) d t=\int_{T}^{\infty}\left(2 P(t)+\frac{R^{\prime}(t)}{R(t)}\right) d t=\int_{T}^{\infty} \frac{2 \beta}{t} d t=\infty .
$$

Proof of Theorem 5.3 We deduce Theorem 5.3 from Theorem 3.1 applied to system (6.51), and by substitution $\xi_{1,2}= \pm i k=$ const $, \alpha=0, \operatorname{Tr}(A)=-2 P, a_{11}=0, a_{12}=1, p_{1}=p_{2} \equiv 0$, $\operatorname{Re}[s]=0$,

$$
\frac{C A_{1}}{\xi_{1}-\xi_{2}}=\frac{C A_{2}}{\xi_{1}-\xi_{2}}=\frac{R(t)-k^{2}}{2 i k}, \quad H A \equiv 0
$$

From (3.5), (3.13)

$$
J_{0}=\left|\frac{R(t)-k^{2}}{k}\right|, \quad J_{1}=\Re\left[2 \theta_{1}\right]+J_{0}=\operatorname{Re}[-2 P]+\left|\frac{R(t)-k^{2}}{k}\right| .
$$


Conditions (3.1), (3.6) turn to (5.6), (5.7). If $R(t)-k^{2} \neq 0$, then (3.2) is satisfied. The case $R(t) \equiv k^{2}$ is trivial, since in this case $C A_{j} \equiv 0$ and the functions $e^{ \pm i k t-\int_{T}^{t} P d s}$ are exact solutions of (2.1).

Proof of Theorem 5.4 We deduce Theorem 5.4 from Theorem 3.4 by choosing

$$
\xi_{1}=i k, \quad \xi_{2}=-i k, \quad k=\text { const }>0, \quad p_{1}=p_{2}=0
$$

From

$$
\xi=i k, \quad \operatorname{Re}[s]=0, \quad C L_{1}=\xi_{1}^{\prime}+\xi_{1}^{2}+R=R-k^{2}, \quad C L_{2}=R-k^{2}, \quad H L \equiv 0,
$$

we get from (3.19)

$$
J_{0}=\left|\frac{C L_{1}}{i k}\right|=\frac{\left|R(t)-k^{2}\right|}{k}
$$

and from (3.18)

$$
J_{3}=\Re\left[\xi_{1}-\xi_{2}-\frac{\xi^{\prime}}{\xi}-2 P\right]=-2 \Re[P(t)]
$$

so (3.2) is satisfied if $\left|R(t)-k^{2}\right|>0$ and condition (3.6) turns to (5.7). Case $R(t)=k^{2}$ is trivial.

Proof of Example 5.2 This example follows from Theorem 5.3.

From $\gamma \leq 1$ we get $|P(t)|=\left|b t^{\gamma-1}\right| \leq C$.

$$
R(t)=Q-P^{2}-P^{\prime}=k^{2}+t^{-\beta}-b^{2} t^{2 \gamma-2}-b(\gamma-1) t^{\gamma-2} .
$$

If $1-\beta<\gamma \leq 1, k b>\frac{1}{2}$, then

$$
2 P-\frac{\left|R-k^{2}\right|}{k}=\frac{2 b k-\left|t^{1-\gamma-\beta}-b^{2} t^{\gamma-1}-b(\gamma-1) t^{-1}\right|}{k} t^{\gamma-1} \geq c t^{\gamma-1} .
$$

If $1-\beta=\gamma \leq 1, k b>\frac{1}{2}$, then

$$
2 P-\frac{\left|R-k^{2}\right|}{k}=\frac{2 b k-\left|1-b^{2} t^{\gamma-1}-b(\gamma-1) t^{-1}\right|}{k} t^{\gamma-1} \geq c t^{\gamma-1} .
$$

If $\gamma=0, \beta \geq 1, b k>\frac{1}{2}$, then

$$
2 P-\frac{\left|R-k^{2}\right|}{k}=\frac{2 b k-\left|t^{1-\beta}+\left(b-b^{2}\right) t^{-1}\right|}{k t} \geq \frac{c}{k t} .
$$

In all these cases, (5.7) is satisfied since $\gamma>0$.

Proof of Theorem 5.5 Theorem 5.5 follows from Theorem 4.2 applied to (6.51). 
Proof of Theorem 5.6 We deduce Theorem 5.6 from Theorem 3.4 by choosing

$$
\xi_{1}=\frac{1}{t}, \quad \xi_{2}=0, \quad p_{1}=p_{2}=0 .
$$

From $t \geq T>0$ we have

$$
C A_{1}=C A_{2}=R(t), \quad H A=0, \quad e_{s}(t)=e_{2 \xi}(t)=\frac{t}{T}, \quad e_{-s}(t)=\frac{T}{t} \leq \frac{t}{T},
$$

and from (3.19) we get

$$
J_{0}(t)=|t R(t)| \sqrt{\left(\left|e_{s}\right|-\left|e_{-s}\right|\right)^{2}+4}=\left|t R(t)\left(\left|e_{s}\right|+\left|e_{-s}\right|\right)\right| \leq \frac{2 t^{2}|R(t)|}{T} .
$$

From (3.18) and (3.6) we get (5.10)

$$
J_{3}(t)=\Re\left[\frac{2}{t}-2 P(t)+J_{0}(t)\right] \leq \Re\left[\frac{2}{t}-2 P(t)+\frac{2 t^{2}|R(t)|}{T}\right] .
$$

Condition (3.2) is satisfied if $R(t) \neq 0$. The case $R(t) \equiv 0$ is obvious since in that case the exact solutions of (2.1) are $u_{1}=t e^{-\int_{T}^{t} P(s, u) d s}, u_{2}=e^{-\int_{T}^{t} P(s, u) d s}$.

Proof of Example 5.3 This example follows from Theorem 5.5:

$$
\begin{aligned}
& P=\frac{a}{t}, \quad Q=\frac{a^{2}-a}{t^{2}}+\frac{b}{t^{3} \ln ^{2}(t)}, \\
& R=Q-P^{\prime}-P^{2}=\frac{b}{t^{3} \ln ^{2}(t)}, \quad t^{2} R(t) \in L_{1}(T, \infty) .
\end{aligned}
$$

Proof of Theorem 5.7 Theorem 5.7 follows from Theorem 3.2 applied to (6.51), and by choosing

$$
\begin{aligned}
& \theta_{1,2}= \pm \sqrt{-R}-\frac{R^{\prime}}{4 R}-P, \quad p_{1}=\xi_{1}-\xi_{2}=2 \xi, \quad p_{2}=0, \\
& \alpha=1, \quad s=0, \quad \xi=\sqrt{-R}, \quad \frac{\xi^{\prime}}{\xi}=\frac{R^{\prime}}{2 R}, \\
& \frac{C L_{1}}{\xi_{1}-\xi_{2}}=\frac{C L_{2}}{\xi_{1}-\xi_{2}}=\frac{5 R^{\prime 2}-4 R^{\prime \prime} R}{32 R^{2} \sqrt{-R}}=\frac{1}{2}\left((-R)^{-1 / 4}\right)^{\prime \prime}(-R)^{-1 / 4}, \\
& H L=0, \quad J_{0}=\left|\frac{C L_{1}+C L_{2}}{\xi_{1}-\xi_{2}}-2 \xi\right|=\left|\left((-R)^{-1 / 4}\right)^{\prime \prime}(-R)^{-1 / 4}-2 \sqrt{-R}\right|, \\
& -J_{1}=2 P-\frac{\xi^{\prime}}{\xi}-J_{0}=2 P-\frac{R^{\prime}}{2 R}-\left|\left((-R)^{-1 / 4}\right)^{\prime \prime}(-R)^{-1 / 4}-2 \sqrt{-R}\right| .
\end{aligned}
$$

For this case (3.2) is true, (3.1) turns to (5.13), and (3.6) turns to (5.14).

Proof of Example 5.4 Example 5.4 follows from Theorem 5.7.

In view of (6.89), we have conditions (5.12), (5.13) of Theorem 5.7 are satisfied if $1 \leq \beta<$ $\gamma$,

$$
R(t)=Q(t)-P^{2}(t)-P^{\prime}(t)=-b^{2} t^{2 \gamma-2}(1+o(1)) \leq 0, \quad t \rightarrow \infty .
$$


Further, in view of

$$
16\left((-R)^{-1 / 4}\right)^{\prime \prime}(-R)^{-1 / 4}=\frac{C+o(1)}{t^{1+\gamma}} \in L_{1}(T, \infty),
$$

condition (5.14) or

$$
\int_{T}^{\infty}\left(P-\frac{R^{\prime}}{4 R}-\sqrt{-R}\right) d t=\infty
$$

is satisfied if $\gamma<2 \beta$ since

$$
\begin{aligned}
P-\sqrt{-R}-\frac{R^{\prime}}{4 R} & =\frac{P^{2}+R}{P+\sqrt{-R}}-\frac{R^{\prime}}{4 R}=\frac{Q-P^{\prime}}{P+\sqrt{-R}}-\frac{R^{\prime}}{4 R} \\
& =\frac{Q(1+o(1))}{2 P}-\frac{\gamma-1+o(1)}{2 t}=\frac{c(1+o(1))}{2 b} t^{2 \beta-\gamma-1}, \quad t \rightarrow \infty .
\end{aligned}
$$

Proof of Example 5.5 Example 5.5 follows from Theorem 5.7. Indeed

$$
P=\frac{n-1}{2 t}, \quad P^{\prime}+P^{2}=\frac{(n-1)(n-3)}{4 t^{2}}, \quad R=\frac{A|u|^{2 \beta}}{1+t^{2}}-\frac{(n-1)(n-3)}{4 t^{2}} .
$$

Choosing

$$
\theta_{1}=\frac{a_{j}}{t}-P(t), \quad \xi_{1}=\frac{a_{j}}{t}, \quad a_{1,2}=\frac{1 \pm \sqrt{1+4(n-1)(n-3)}}{2}, \quad u=e^{\int_{T}^{t} \theta_{1} d s},
$$

we get

$$
R=\frac{A e^{\int_{T}^{t} \Re\left[2 \beta \theta_{1}\right] d s}}{1+t^{2}}-\frac{(n-1)(n-3)}{4 t^{2}}=\frac{C t^{\beta\left(2 a_{1}+1-n\right)}}{1+t^{2}}-\frac{(n-1)(n-3)}{4 t^{2}} .
$$

If $n>3, \beta>0$, then

$$
\beta\left(n-1-2 a_{1}\right)<0
$$

or

$$
(n-2)^{2}-1-4(n-1)(n-3)=-3(n-3)(n-1)<0,
$$

and conditions (5.12), (5.13) are satisfied:

$$
R(t)=\frac{o(1)-(n-1)(n-3)}{4 t^{2}}<0, \quad \frac{R^{\prime}(t)}{4 R(t)}=\frac{o(1)-2}{t} .
$$

From

$$
\frac{5 R^{\prime 2}}{R^{2} \sqrt{-R}}-\frac{4 R^{\prime \prime}}{R \sqrt{-R}}=\frac{C+o(1)}{t^{2}} \in L_{1}(T, \infty)
$$

condition (5.14) is satisfied since

$$
P-\frac{R^{\prime}}{4 R}-\sqrt{-R}=\frac{o(1)+n-\sqrt{(n-1)(n-3)}}{2 t}=\frac{o(1)+4 n-3}{2 t(n+\sqrt{(n-1)(n-3)})} \geq \frac{C}{t} .
$$


Proof of Theorem 5.8 We deduce Theorem 5.8 from Theorem 3.4 by choosing $p_{1}(t)=$ $p_{2}(t)=0$, the phase $\xi_{2}(t)$ from the Hartman-Wintner approximation

$$
\xi_{2}(t)=-i \sqrt{R\left(1-r^{2}\right)}-\frac{R^{\prime}}{4 R}
$$

and $\xi_{1}(t)$ from $(6.57)$

$$
\xi_{1}(t)-\xi_{2}(t)=\frac{d}{d t} \ln \left(1+2 \xi(T) \int_{T}^{t} e^{-\int_{T}^{s} 2 \xi_{2} d y} d s\right)
$$

or $(5.20)$

$$
2 \xi(t)=\xi_{1}(t)-\xi_{2}(t)=\frac{d}{d t} \ln \left(1+2 \xi_{0} \int_{T}^{t}|R(s) / R(T)|^{1 / 2} e^{2 i \int_{T}^{s} \sqrt{R\left(1-r^{2}\right)} d y} d s\right) .
$$

Since $2 \xi+2 \xi_{2}+\frac{\xi^{\prime}}{\xi}=0$, we get, from (3.18), $J_{3}=J_{0}+\Re\left(2 \xi-\frac{\xi^{\prime}}{\xi}-2 P\right)$

$$
J_{3}=J_{0}+\Re\left(4 \xi+2 \xi_{2}-2 P\right)=J_{0}+\Re\left[-2 i \sqrt{R\left(1-r^{2}\right)}-\frac{R^{\prime}}{2 R}+4 \xi-2 P\right] .
$$

Condition (3.6) with $J=J_{3}$ turns to (5.18). From (3.32) in view of (6.90), we get (5.19):

$$
J_{0}(t)=\left|\left(\sqrt{r^{2}-1}-r\right)^{\prime}\right|\left|\frac{1+\left|1+2 \xi(T) \int_{T}^{t} \sqrt{|R(s)|} e^{2 i \int_{T}^{s} 2 \sqrt{R\left(1-r^{2}\right)} d y} d s\right|^{2}}{2|\xi(T)|}\right| .
$$

Condition (3.2) is satisfied in view of Remark 3.3 and $J_{0}(t)>0$.

Proof of Theorem 5.9 We deduce Theorem 5.9 from Theorem 3.8 by choosing

$$
\xi_{2}=-i \sqrt{R(t)}-\frac{R^{\prime}}{4 R}
$$

By calculations

$$
\xi_{2}^{\prime}(t)+\xi_{2}^{2}(t)+R(t)=\left(R^{-1 / 4}\right)^{\prime \prime} R^{1 / 4}
$$

we get (5.22) from (3.29). Further, from (3.33) we get (5.23) since

$$
2 P-2 \xi_{2}-4 \xi-J_{0}=2 P+\frac{R^{\prime}}{2 R}+2 i \sqrt{R(t)}-4 \xi-J_{0}
$$

Proof of Theorem 5.10 We deduce Theorem 5.10 from Theorem 3.4 by taking

$$
\xi_{1}(t)=P(t)-S(t), \quad S(t)=\int_{T}^{t} Q(s) e^{-\int_{s}^{t} 2 P(y) d y} d s
$$

and assuming $H L \equiv 0$ or $\xi_{1}+\xi_{2}+\frac{\xi^{\prime}}{\xi}=0$.

Since $S^{\prime}(t)+2 P(t) S(t)-Q(t)=0, C L_{j}=\xi_{j}^{\prime}(t)+\xi_{j}^{2}(t)+R(t)$, we get

$$
C L_{2}=C L_{1}=\xi_{1}^{\prime}(t)+\xi_{1}^{2}(t)+R(t)=P^{\prime}-S^{\prime}+P^{2}-2 P S+S^{2}+Q-P^{2}-P^{\prime}=S^{2}(t) .
$$


From (3.33) and $2 P-2 \xi_{1}-J_{0}=2 S-J_{0}$, we get

$$
\int_{T}^{t} \Re\left[2 S(t)-J_{0}(t)\right] d t=\infty
$$

where in view of $\xi_{1}+\xi_{2}+\frac{\xi^{\prime}}{\xi}=0$ we have

$$
\begin{aligned}
& \xi(t)=-\frac{1}{2} \frac{d}{d t} \ln \left|\int_{t}^{\infty} e^{\int_{T}^{s}(2 S-2 P) d y} d s\right|=\frac{e^{\int_{T}^{t}(2 S-2 P) d y}}{2 \int_{t}^{\infty} e^{\int_{T}^{s}(2 S-2 P) d y} d s}>0, \\
& e_{-2 \xi}(t)=\frac{\int_{t}^{\infty} e^{\int_{T}^{s}(2 S-2 P) d y} d s}{\int_{T}^{\infty} e^{\int_{T}^{s}(2 S-2 P) d y} d s} \leq 1 .
\end{aligned}
$$

Further from (3.15)

$$
\begin{aligned}
J_{0}(t) & =\left|\frac{C L_{1}\left(e_{2 \xi}+e_{-2 \xi}\right)}{2 \xi}\right|=\frac{\int_{t}^{\infty} e^{\int_{T}^{s}(2 S-2 P) d y} d s}{e^{\int_{T}^{t}(2 S-2 P) d y}}\left(e_{2 \xi}(t)+e_{-2 \xi}\right) S^{2}(t) \\
& =\frac{\int_{t}^{\infty} e^{\int_{T}^{s}(2 S-2 P) d y} d s}{e^{\int_{T}^{t}(2 S-2 P) d y}}\left(\frac{\int_{T}^{\infty} e^{\int_{T}^{s}(2 S-2 P) d y} d s}{\int_{t}^{\infty} e^{\int_{T}^{S}(2 S-2 P) d y} d s}+\frac{\int_{t}^{\infty} e^{\int_{T}^{s}(2 S-2 P) d y} d s}{\int_{T}^{\infty} e^{\int_{T}^{s}(2 S-2 P) d y} d s}\right) S^{2}(t) \\
& \leq 2 e^{\int_{T}^{t}(2 P-2 S) d y} S^{2}(t) \int_{T}^{\infty} e^{\int_{T}^{s}(2 S-2 P) d y} d s=2 S^{2}(t) \int_{T}^{\infty} e^{\int_{t}^{s}(2 S-2 P) d y} d s,
\end{aligned}
$$

and (3.6) turns to (5.28).

\section{Appendix: Some invariants of the planar dynamic systems}

By a linear time-dependent non-singular lower triangular transformation

$$
v(t)=S(t) u(t), \quad S(t)=\left(\begin{array}{cc}
s_{11}(t) & 0 \\
s_{21}(t) & s_{22}(t)
\end{array}\right)
$$

from linear system $(1.1)(A(t)$ does not depend on $u(t))$, we get another linear system

$$
v^{\prime}(t)=B(t) v(t), \quad B(t)=S(t) A(t) S^{-1}(t)+S^{\prime}(t) S^{-1}(t) .
$$

Define auxiliary functions associated with system (A.2) that depend on phase functions $\eta_{j}(t)$ as follows:

$$
\begin{aligned}
& C B_{j}(t)=\eta_{j}^{\prime}(t)+\eta_{j}^{2}(t)-\eta_{j}(t)\left[\operatorname{Tr}(B(t))+\frac{b_{12}^{\prime}(t)}{b_{12}(t)}\right]+\operatorname{det}(B(t))+\frac{W\left[b_{11}, b_{12}\right]}{b_{12}(t)}, \\
& H B(t)=\frac{C B\left(\eta_{1}\right)-C B\left(\eta_{2}\right)}{\eta_{1}(t)-\eta_{2}(t)}=\eta_{1}(t)+\eta_{2}(t)-\operatorname{Tr}(B(t))+\left(\ln \left|\frac{\eta_{1}(t)-\eta_{2}(t)}{b_{12}(t)}\right|\right)^{\prime}, \\
& R B(t)=\operatorname{det}(B(t))-\frac{(\operatorname{Tr} B(t))^{2}}{4}+\frac{W\left[b_{11}-b_{22}, b_{12}(t)\right]}{2 b_{12}(t)}-\left(b_{12}^{-1 / 2}\right)^{\prime \prime}(t) b_{12}^{1 / 2}(t),
\end{aligned}
$$

where $\eta_{j}(t)$ are the phase functions of system (A.2). 
Theorem A.1 Assume that $A(t) \in C^{2}(T, \infty), B(t) \in C^{2}(T, \infty)$, and $S(t) \in C^{4}(T, \infty)$ is a non-singular lower triangular transformation, and $\theta_{j}(t), \eta_{j}(t)$ are solutions of the characteristic equations of linear systems (1.1), (A.2)

$$
C A\left(\theta_{j}\right)=0, \quad C B\left(\eta_{j}\right)=0, \quad j=1,2
$$

with the initial values

$$
\eta_{j}(T)=\theta_{j}(T)+\frac{s_{11}^{\prime}(T)}{s_{11}(T)}, \quad j=1,2
$$

Then we have the invariance

$$
\begin{aligned}
& R B(t)=R A(t), \quad R B^{\prime}(t)=R A^{\prime}(t), \\
& \theta_{1}-\theta_{2}=\eta_{1}-\eta_{2}, \quad \frac{R A^{\prime}(t)}{2 R A(t)}-\operatorname{Tr}(A)=\frac{R B^{\prime}(t)}{2 R B(t)}-\operatorname{Tr}(B)+\frac{(\operatorname{det}(S))^{\prime}}{\operatorname{det}(S)} .
\end{aligned}
$$

Remark A.1 From Theorem A.1 it follows the well-known result that the function

$$
R(t)=Q(t)-P^{2}(t)-P^{\prime}(t)
$$

is invariant of (1.6) with respect to the transformation $V(t)=v(t) w(t)$.

Proof of Theorem A.1 By substitution

$$
\xi_{j}(t)=\theta_{j}(t)-\frac{\operatorname{Tr}(A(t))}{2}-\frac{a_{12}^{\prime}(t)}{2 a_{12}(t)}, \quad \zeta_{j}(t)=\eta_{j}(t)-\frac{\operatorname{Tr}(B(t))}{2}-\frac{b_{12}^{\prime}(t)}{2 b_{12}(t)}
$$

we get from $(2.8)$

$$
C A_{j}(t)=\xi_{j}^{\prime}(t)+\xi_{j}^{2}(t)+R A(t)=0, \quad C B_{j}(t)=\zeta_{j}^{\prime}(t)+\zeta_{j}^{2}(t)+R B(t)=0,
$$

where $R A(t), R B(t)$ are defined in (2.10), (A.5).

By direct calculations, from (A.2) we get

$$
\begin{aligned}
& b_{12}(t)=\frac{a_{12}(t) s_{11}(t)}{s_{22}(t)}, \quad b_{11}=a_{11}+\frac{s_{11}^{\prime}}{s_{11}}-\frac{s_{21} a_{12}}{s_{22}}, \quad b_{22}=a_{22}+\frac{s_{21} a_{12}}{s_{22}}+\frac{s_{22}^{\prime}}{s_{22}}, \\
& b_{21}=\frac{s_{21}}{s_{11}}\left(a_{11}-a_{22}-\frac{s_{21} a_{12}}{s_{22}}-\frac{s_{22}^{\prime}}{s_{22}}\right)+\frac{s_{22} a_{21}}{s_{11}}+\frac{s_{21}^{\prime}}{s_{11}}
\end{aligned}
$$

and (A.8). Further, we get $C A_{j}(t)-C B_{j}(t)=0$, or

$$
\left(\xi_{j}-\zeta_{j}\right)^{\prime}(t)+\left(\xi_{j}-\zeta_{j}\right)\left(\xi_{j}+\zeta_{j}\right)=0, \quad\left(\xi_{j}-\zeta_{j}\right)=C_{j} e^{-\int_{T}^{t}\left(\xi_{j}+\zeta_{j}\right) d s}
$$

In view of $b_{12}=\frac{a_{12} s_{11}}{s_{22}}$, and

$$
\operatorname{Tr}(B)=\operatorname{Tr}(A)+\frac{(\operatorname{det}(S))^{\prime}}{\operatorname{det}(S)},
$$


assuming initial conditions (A.7), we get

$$
\left(\xi_{j}-\zeta_{j}\right)(T)=\left(\theta_{j}-\eta_{j}\right)(T)+\frac{s_{11}^{\prime}(T)}{s_{11}(T)}=0, \quad C_{j} \equiv 0, \quad \xi_{j}(t)=\zeta_{j}(t), \quad j=1,2 .
$$

So, the solutions $\theta_{j}, \eta_{j}$ of characteristic equations $C A_{j}=C B_{j}=0$ are connected:

$$
\theta_{j}(t)-\frac{\operatorname{Tr} A(t)}{2}-\frac{a_{12}^{\prime}(t)}{2 a_{12}(t)}=\eta_{j}(t)-\frac{\operatorname{Tr} B(t)}{2}-\frac{b_{12}^{\prime}(t)}{2 b_{12}(t)}, \quad j=1,2 .
$$

From these expressions, we get (A.9).

Proof of Remark A.1 Rewrite equation (2.1) in form (6.6). Choosing

$$
S=\left(\begin{array}{cc}
w(t) & 0 \\
w^{\prime}(t) & w(t)
\end{array}\right),
$$

we have

$$
B=S A S^{-1}+S^{\prime} S^{-1}=\left(\begin{array}{cc}
0 & 1 \\
-\hat{Q}(t) & -2 \hat{P}(t)
\end{array}\right),
$$

where

$$
\begin{aligned}
& \hat{P}(t)=P(t)-\frac{w^{\prime}(t)}{w(t)}, \quad \hat{Q}(t)=Q(t)-\frac{2 w^{\prime}(t) P(t)}{w(t)}-\frac{w^{\prime \prime}(t)}{w(t)}+\frac{2 w^{\prime 2}(t)}{w^{2}(t)}, \\
& \hat{R}(t) \equiv \hat{Q}(t)-\hat{P}^{2}(t)-\hat{P}^{\prime}(t),
\end{aligned}
$$

and (A.8) becomes $\hat{R}(t)=R(t)$.

Remark A.2 There are several characteristic functions of (1.1) depending on the structure of the matrix $A(t)$. Indeed, if $a_{12}(t) \neq 0$, then the characteristic function $C A_{j}(t)$ of $(1.1)$ is given by (2.8). If $a_{12} \equiv 0$, but $a_{21} \neq 0$, the characteristic function may be defined by the similar formula

$$
C C A_{j}(t)=\theta_{j}^{\prime}(t)+\theta_{j}^{2}(t)-\theta_{j}(t)\left[\operatorname{Tr}(A)+\frac{a_{21}^{\prime}(t)}{a_{21}(t)}\right]+\operatorname{det}(A(t))+\frac{W\left[a_{22}, a_{21}\right]}{a_{21}(t)} .
$$

If system (1.1) is diagonal, that is, $a_{12}(t) \equiv a_{21}(t) \equiv 0$, then

$$
C A_{1}(t)=a_{11}(t)-\theta_{1}(t)=0, \quad C A_{2}(t)=a_{11}(t)-\theta_{2}(t)=0 .
$$

Competing interests

The author declares that he has no competing interests.

\section{Acknowledgements}

This paper is dedicated to my mother Paytsar Hovhannisyan.

The author would like to thank anonymous reviewers for very useful and constructive comments that helped to improve the original manuscript. 


\section{References}

1. Cesary, L: Asymptotic Behavior and Stability Problems in Ordinary Differential, 3rd edn. Springer, Berlin (1970)

2. Lakshmikantham, V, Leela, S, Martynuk, AA: Stability Analysis of Nonlinear Systems. Dekker, New York (1989)

3. Lakshmikantham, V, Matrosov, VM, Sivasundaram, S: Vector Lyapunov Functions and Stability Analysis of Nonlinear Systems. Math. and Its Appl., vol. 63. Kluwer Academic, Dordrecht (1991)

4. Hovhannisyan, GR: Asymptotic stability for second-order differential equations with complex coefficients. Electron. J. Differ. Equ. 2004, 85 (2004)

5. Hovhannisyan, GR: Asymptotic stability and asymptotic solutions of second-order differential equations. J. Math. Anal. Appl. 327, 47-62 (2007)

6. Hovhannisyan, GR: Levinson theorem for $2 \times 2$ system and applications to the asymptotic stability and Schrodinger equation. Int. J. Evol. Equ. 3(2), 181-203 (2007)

7. Hovhannisyan, GR, Liu, W: On nonautonomous Dirac equation. J. Math. Phys. 50(12), 123507 (2009)

8. Matukuma, T: Sur la dynamique des amas globulaires stellaires. Proc. Imp. Acad. (Tokyo) 6, 133-136 (1930)

9. Pucci, P, Serrin, J: Asymptotic stability for ordinary differential systems with time dependent restoring potentials. Arch Ration. Mech. Anal. 113, 1-32 (1995)

10. Ignatyev, AO: Stability of a linear oscillator with variable parameters. Electron. J. Differ. Equ. 1997, 17 (1997)

11. Duc, LH, Ilchmann, A, Siegmund, S, Taraba, P: On stability of linear time-varying second-order differential equations. Q. Appl. Math. 64, 137-151 (2006)

12. Ballieu, RJ, Peiffer, K: Asymptotic stability of the origin for the equation $x^{\prime \prime}(t)+f\left(t, x, x^{\prime}(t)\right)\left|x^{\prime}(t)\right|^{\alpha}+g(x)=0$. J. Math. Anal. Appl. 34, 321-332 (1978)

13. Smith, RA: Asymptotic stability of $x^{\prime \prime}+a(t) x^{\prime}+x=0$. Q. J. Math. 12, 123-126 (1961)

14. Hartman, P: Ordinary Differential Equations. Wiley, New York (1973)

15. Harris, WA, Pucci, P, Serrin, J: Asymptotic behavior of solutions of a non-standard second order equations. Differ. Integral Equ. 6, 1201-1215 (1993). ISSN:0893-4983

16. Harris, WA, Pucci, P, Serrin, J: Asymptotic estimates for a nonstandard second order differential equation. In: Elworthy, KD, Everitt, WN, Lee, EB (eds.) Differential Equations, Dynamical Systems, and Control Science: A Festschrift in Honor of Lawrence Markus. Lecture Notes in Pure and Appl. Math., pp. 75-85. Dekker, New York (1994)

17. Arstein, Z, Infante, EF: On asymptotic stability of oscillators with unbounded damping. Q. Appl. Mech. 34, 195-198 (1976)

18. Hatvani, L: Integral conditions on asymptotic stability for the damped linear oscillator with small damping. Proc. Am. Math. Soc. 124(2), 415-422 (1996)

19. Levin, JJ, Nobel, JA: Global asymptotic stability of nonlinear systems of differential equations to reactor dynamics. Arch. Ration. Mech. Anal. 5, 104-211 (1960)

20. Pucci, P, Serrin, J: Precise damping conditions for global asymptotic stability for nonlinear second order systems. Acta Math. 170, 275-307 (1993)

21. Salvadori, L: Famiglie ad un parametro di funzioni di Liapunov nello studio della stabilita. In: Symposia Matematica, vol. 4, pp. 307-330. Ist. Naz. di Alta Matematica, Bologna (1971)

doi:10.1186/1687-1847-2013-144

Cite this article as: Hovhannisyan: Stability of planar nonautonomous dynamic systems. Advances in Difference Equations 2013 2013:144.

\section{Submit your manuscript to a SpringerOpen ${ }^{\circ}$ journal and benefit from:}

- Convenient online submission

- Rigorous peer review

- Immediate publication on acceptance

- Open access: articles freely available online

- High visibility within the field

- Retaining the copyright to your article 